\title{
Ring Expansive Routes to Quinolizidine Alkaloids: Formal Synthesis of (-)-
}

\section{Lasubine II}

\author{
Vijaya Gracias, Yibin Zeng, Pankaj Desai, and Jeffrey Aubé* \\ Department of Medicinal Chemistry, 1251 Wescoe Hall Drive, Room 4070, Malott Hall, \\ University of Kansas, Lawrence, Kansas 66045-7582 \\ jaube@ku.edu
}

\section{Supporting Information}

Experimental Details

Copies of ${ }^{1} \mathrm{H}$ and ${ }^{13} \mathrm{C}$ spectra of listed compounds

$\mathrm{GC}$ analysis of

a $1: 2$ mixture of $\mathbf{3 a}$ and $\mathbf{3 b}$

and

the crude nitrone photolysis product (3a) vs (3b)

\section{Corresponding author:}

Professor Jeffrey Aubé

Department of Medicinal Chemistry

1251 Wescoe Hall Drive, Room 4070

Malott Hall

University of Kansas

Lawrence, Kansas 66045-2506

Tel 785.864.4496

Fax 785.864.5326

E-mail: jaube@ku.edu 


\section{Experimental Details}

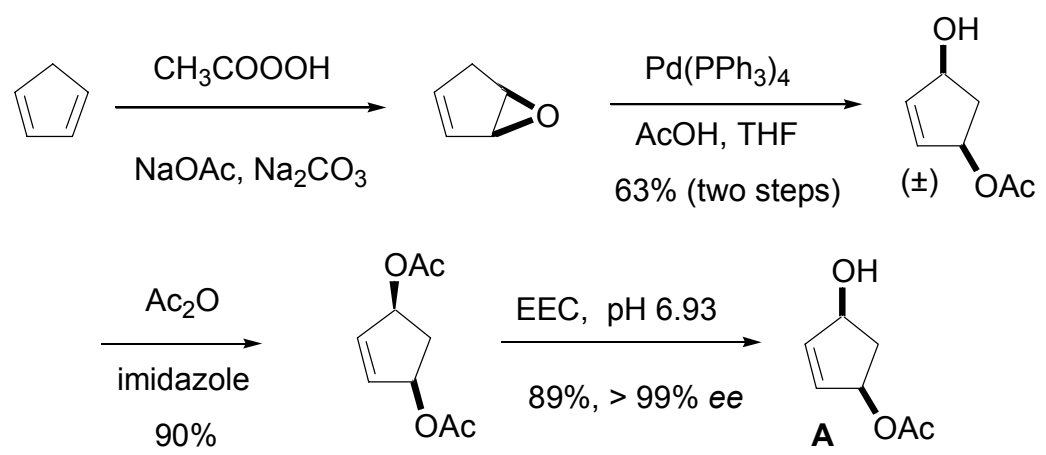

$(1 R, 3 S)-(+)-4-C y c l o p e n t e n e-1,3-d i o l-1-a c e t a t e(A) . ~(1 R, 3 S)-(+)-4-c y c l o p e n t e n e-$ 1,3-diol-1-acetate (A) was prepared from the freshly cracked cyclopentadiene according to literature procedures ${ }^{1-3}$ as white colorless needles. Mp $49-51{ }^{\circ} \mathrm{C}$ (lit. ${ }^{3} 49-51^{\circ} \mathrm{C}$ ); $[\alpha]_{\mathrm{D}}$ $+71.3\left(c\right.$ 1.25, $\left.\mathrm{CHCl}_{3}\right) ;[\alpha]_{\mathrm{D}}+67.5\left(\right.$ c 1.64, $\left.\mathrm{CHCl}_{3}\right)$; Lit. values $[\alpha]_{\mathrm{D}}+73.8$ (c 1.25, $\left.\mathrm{CHCl}_{3}\right){ }^{3}[\alpha]_{\mathrm{D}}+68.0\left(c 1.64, \mathrm{CHCl}_{3}\right) .{ }^{4}$

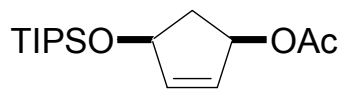

B

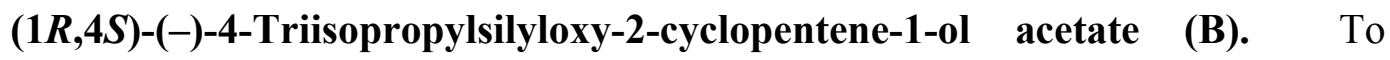
$(1 R, 3 S)-(+)-4$-cyclopentene-1,3-diol-1-acetate $(\mathbf{A})(3.68 \mathrm{~g}, 25.9 \mathrm{mmol})$ in DMF $(20 \mathrm{~mL})$ was added imidazole $(5.28 \mathrm{~g}, 77.7 \mathrm{mmol})$ in one portion. Triisopropylsilylchloride (16.5 $\mathrm{mL}, 77.7 \mathrm{mmol}$ ) was added to the reaction mixture, which was stirred at room temperature under argon for $13 \mathrm{~h}$. The reaction mixture was diluted with $\mathrm{CH}_{2} \mathrm{Cl}_{2}(200$ $\mathrm{mL})$ and the organic layer was washed with $2 \mathrm{~N}$ sulfuric acid $(50 \mathrm{~mL}), \mathrm{H}_{2} \mathrm{O}(25 \mathrm{~mL})$, and brine $(25 \mathrm{~mL})$. The organic layer was dried (anhyd $\left.\mathrm{Na}_{2} \mathrm{SO}_{4}\right)$ and concentrated to afford a clear oil. Flash chromatography (25\% EtOAc/hexane) gave $5.5 \mathrm{~g}(72 \%)$ of $\mathbf{B}$ as a clear 
oil. $\mathrm{R}_{f}=0.6(10 \%$ EtOAc/hexane $) ;{ }^{1} \mathrm{H}$ NMR $\left(400 \mathrm{MHz}, \mathrm{CDCl}_{3}\right) \delta 1.04-1.14(\mathrm{~m}, 21 \mathrm{H})$, $1.64(\mathrm{dt}, J=5.1,13.7 \mathrm{~Hz}, 1 \mathrm{H}), 2.04(\mathrm{~s}, 3 \mathrm{H}), 2.84(\mathrm{dt}, J=7.3,14.5 \mathrm{~Hz}, 1 \mathrm{H}), 4.79(\mathrm{~m}, 1 \mathrm{H})$, $5.46(\mathrm{~m}, 1 \mathrm{H}), 5.88(\mathrm{dt}, J=1.6,5.6 \mathrm{~Hz}, 1 \mathrm{H}), 6.02(\mathrm{dt}, J=1.6,5.6 \mathrm{~Hz}, 1 \mathrm{H}) ;{ }^{13} \mathrm{C} \mathrm{NMR}$ $\left(100.6 \mathrm{MHz}, \mathrm{CDCl}_{3}\right) \delta 12.1,17.8,17.9,21.1,41.5,74.8,76.8,131.0,139.0,170.8 ; \mathrm{IR}$ (neat) 2946, 2866, $1738 \mathrm{~cm}^{-1}$; MS (CI) $\mathrm{m} / z 299\left(\mathrm{M}^{+}+1\right), 239,125$; HRMS calcd for $\mathrm{C}_{16} \mathrm{H}_{31} \mathrm{O}_{3} \mathrm{Si}\left(\mathrm{M}^{+}+1\right): 299.2042$, found 299.2057; $[\alpha]_{\mathrm{D}}-6.33$ (c 1.53, MeOH).

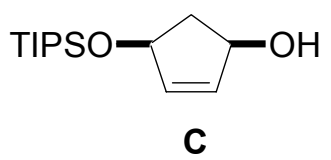

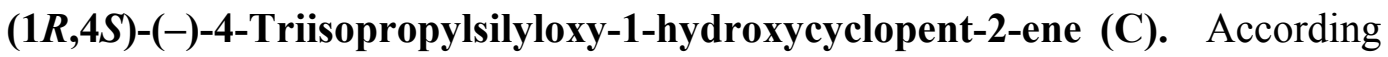
to the procedure of Paquette et al., ${ }^{5}$ to $(1 R, 4 S)-(-)-4$-triisopropylsilyloxy-2cyclopentenylacetate $(\mathbf{B})(6.00 \mathrm{~g}, 20.1 \mathrm{mmoL})$ in $\mathrm{CH}_{3} \mathrm{OH}(100 \mathrm{~mL})$ was added sodium methoxide $(2.17 \mathrm{~g}, 40.2 \mathrm{mmol})$ in one portion. The reaction mixture was stirred at room temperature under argon for $15 \mathrm{~min}$, after which time it was concentrated and diluted with $\mathrm{CH}_{2} \mathrm{Cl}_{2}(100 \mathrm{~mL})$. The organic layer was washed with water $(10 \mathrm{~mL})$, dried (anhyd $\left.\mathrm{Na}_{2} \mathrm{SO}_{4}\right)$ and concentrated to give a clear oil. Flash chromatography $(10 \%$ EtOAc/hexane) afforded $4 \mathrm{~g}(77 \%)$ of $\mathbf{C}$ as a clear oil. $\mathrm{R}_{f}=0.4\left(10 \%\right.$ EtOAc/hexane). ${ }^{1} \mathrm{H}$ NMR (400 MHz, $\left.\mathrm{CDCl}_{3}\right) \delta 1.04-1.14(\mathrm{~m}, 21 \mathrm{H}), 1.57(\mathrm{dt}, J=4.5,13.7 \mathrm{~Hz}, 1 \mathrm{H}), 1.61(\mathrm{br}$ s, 1H), $2.71(\mathrm{dt}, J=6.9,13.7 \mathrm{~Hz}, 1 \mathrm{H}), 4.59(\mathrm{~m}, 1 \mathrm{H}), 4.74(\mathrm{~m}, 1 \mathrm{H}), 5.94(\mathrm{~m}, 2 \mathrm{H}) ;{ }^{13} \mathrm{C}$ NMR (100.6 MHz, $\left.\mathrm{CDCl}_{3}\right) \delta 12.1,18.0,45.0,75.1,75.2,135.5,137.2$; IR (neat) 3400 $\mathrm{cm}^{-1}$; MS (CI) $m / z 257\left(\mathrm{M}^{+}+1\right), 239$; HRMS calcd for $\mathrm{C}_{14} \mathrm{H}_{29} \mathrm{O}_{2} \mathrm{Si}\left(\mathrm{M}^{+}+1\right)$ : 257.1937, found 257.1923; $[\alpha]_{\mathrm{D}}-19.4($ c $0.50, \mathrm{MeOH})$. 


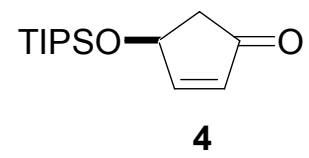

(4S)-(-)-4-Triisopropylsilyloxy-2-cyclopenten-1-one (4). According to the procedure of Paquette et al., ${ }^{5}$ to $(1 R, 4 S)-(-)-4$-triisopropylsiloxy-1-hydroxycyclopent-2ene $(\mathbf{C})(4.00 \mathrm{~g}, 15.6 \mathrm{mmoL})$ in $\mathrm{CH}_{2} \mathrm{Cl}_{2}(20 \mathrm{~mL})$ was added activated $\mathrm{MnO}_{2}(9.40 \mathrm{~g}$, $109.3 \mathrm{mmoL}$ ). The resulting suspension was stirred under argon. After $3 \mathrm{~h}$, additional manganese dioxide (4.69 g, $54.6 \mathrm{mmoL})$ was added and stirring was continued for another $12 \mathrm{~h}$. The reaction mixture was vacuum filtered through a pad of Celite, and the pad was washed with $\mathrm{CH}_{2} \mathrm{Cl}_{2}(200 \mathrm{~mL})$. The organic layer was concentrated and purified by flash chromatography (10\% EtOAc/hexane) to give $3.29 \mathrm{~g}$ of $4(83 \%)$ as a clear oil. $\mathrm{R}_{f}=0.5\left(10 \%\right.$ EtOAc/hexane). ${ }^{1} \mathrm{H}$ NMR $\left(400 \mathrm{MHz}, \mathrm{CDCl}_{3}\right) \delta$ 1.05-1.16 (m, 21H), $2.30(\mathrm{dd}, J=2.3,18.1 \mathrm{~Hz}, 1 \mathrm{H}), 2.74(\mathrm{dd}, J=5.9,18.1 \mathrm{~Hz}, 1 \mathrm{H}), 5.07(\mathrm{~m}, 1 \mathrm{H}), 6.18$ $(\mathrm{dd}, J=1.2,5.6 \mathrm{~Hz}, 1 \mathrm{H}), 7.50(\mathrm{dd}, J=2.2,5.6 \mathrm{~Hz}, 1 \mathrm{H}) ;{ }^{13} \mathrm{C} \mathrm{NMR}\left(100.6 \mathrm{MHz}, \mathrm{CDCl}_{3}\right)$ $\delta 12.0,17.8$ (2 carbons), 45.3, 70.9, 134.3, 163.8, 206.4; IR (neat) 2947, 2866, $1716 \mathrm{~cm}^{-}$ 1; MS (CI) m/z $255\left(\mathrm{M}^{+}+1\right), 211$; HRMS calcd for $\mathrm{C}_{14} \mathrm{H}_{27} \mathrm{O}_{2} \mathrm{Si}\left(\mathrm{M}^{+}+1\right): 255.1780$, found $255.1804 ;[\alpha]_{\mathrm{D}}-58.8(c$ 1.05, $\mathrm{MeOH})$.

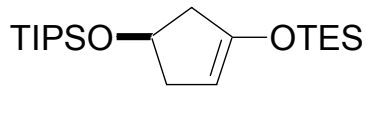

5

(4R)-(+)-1-Triethylsiloxy-4-triisopropylsiloxy-cyclopentene (5). According to the procedure of Johnson et al., ${ }^{6}$ to triethylsilane $(2.14 \mathrm{~mL}, 13.4 \mathrm{mmol})$ was added 15 
drops of Karstedt's catalyst. The reaction mixture was stirred under argon at room temperature for $15 \mathrm{~min}$. After this time (4S)-(-)-4-triisopropylsilyloxy-2-cyclopenten-1one (4) (1.71 g, $6.73 \mathrm{mmol})$ was added and the reaction mixture was heated on an oil bath at $100{ }^{\circ} \mathrm{C}$ for $1 \mathrm{~h}$. The reaction mixture was cooled to room temperature and stirred for an additional $15 \mathrm{~h}$. Flash chromatography (2\% EtOAc/hexane) gave $2.4 \mathrm{~g}(96 \%)$ of 5 as a tan oil. $\mathrm{R}_{f}=0.7\left(2 \%\right.$ EtOAc/hexane). ${ }^{1} \mathrm{H}$ NMR (400 $\left.\mathrm{MHz}, \mathrm{CDCl}_{3}\right) \delta 0.67(\mathrm{q}, J=7.9$ $\mathrm{Hz}, 6 \mathrm{H}), 0.97(\mathrm{t}, J=7.9 \mathrm{~Hz}, 9 \mathrm{H}), 1.03-1.06(\mathrm{~m}, 21 \mathrm{H}), 2.21-2.34(\mathrm{~m}, 2 \mathrm{H}), 2.50-2.60(\mathrm{~m}$, 2H), 4.50-4.55 (m, 2H); ${ }^{13} \mathrm{C}$ NMR (100.6 MHz, $\left.\mathrm{CDCl}_{3}\right) \delta$ 4.8, 6.6, 12.1, 17.9, 39.8, 43.9, 70.6, 99.6, 151.9; IR (neat) 2943, 2866, $1644 \mathrm{~cm}^{-1}$; MS (CI) $m / z 371\left(\mathrm{M}^{+}+1\right), 327,132$; HRMS calcd for $\mathrm{C}_{20} \mathrm{H}_{43} \mathrm{O}_{2} \mathrm{Si}\left(\mathrm{M}^{+}+1\right)$ : 371.2812, found 371.2802; $[\alpha]_{\mathrm{D}}+5.02$ (c 5.33, $\left.\mathrm{CHCl}_{3}\right)$.

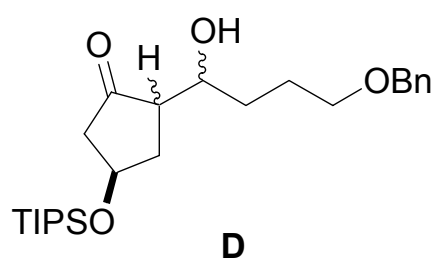

\section{(3R)-5-(4'-Benzyloxy-1'-hydroxybutyl)-3-triisopropylsiloxycyclopentanone}

(D, mixture of isomers). According to the procedure of Danishefsky et al., ${ }^{7}$ to the aldehyde $6^{8}(2.53 \mathrm{~g}, 14.5 \mathrm{mmol})$ in $\mathrm{CH}_{2} \mathrm{Cl}_{2}(40 \mathrm{~mL})$ at $-78{ }^{\circ} \mathrm{C}$ was added freshly distilled $\mathrm{TiCl}_{4}(1.55 \mathrm{~mL}, 14.5 \mathrm{mmol})$ over $20 \mathrm{~min}$. The orange reaction mixture was stirred at -78 ${ }^{\circ} \mathrm{C}$ for an additonal $15 \mathrm{~min}$. After this time the enol ether 5 (2.69 $\left.\mathrm{g}, 7.27 \mathrm{mmol}\right)$ in $\mathrm{CH}_{2} \mathrm{Cl}_{2}(20 \mathrm{~mL})$ was added via syringe pump over $1 \mathrm{~h}$ at $-78^{\circ} \mathrm{C}$. The reaction mixture is stirred for an additional $20 \mathrm{~min}$ and $\mathrm{H}_{2} \mathrm{O}(20 \mathrm{~mL})$ was added rapidly. The reaction mixture was extracted into $\mathrm{CH}_{2} \mathrm{Cl}_{2}(500 \mathrm{~mL})$, dried (anhyd $\left.\mathrm{Na}_{2} \mathrm{SO}_{4}\right)$ and concentrated to 
afford a pale pink oil. Flash chromatography (10\% EtOAc/hexane) gave $2.2 \mathrm{~g}(70 \%)$ of D as a pale yellow oil. The product was obtained as mixture of four diastereomers and was used without further purification. IR (neat) 3444, 2941, $1736 \mathrm{~cm}^{-1}$; MS (CI) $\mathrm{m} / z 435$ $\left(\mathrm{M}^{+}+1\right), 257,243,179,108,91,71$; HRMS calcd for $\mathrm{C}_{25} \mathrm{H}_{43} \mathrm{SiO}_{4}\left(\mathrm{M}^{+}+1\right)$ : 435.2931, found 435.2894 .

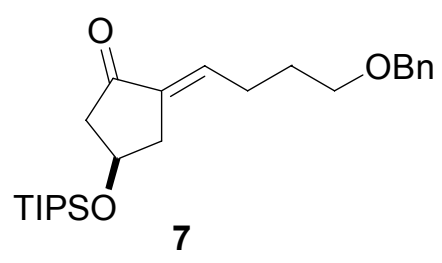

(3R)-(+)-5-(4'-Benzyloxybutylidene)-3-triisopropylsiloxy-cycopentanone (7).

To a solution of keto alcohol diastereomers $\mathbf{D}(1.1 \mathrm{~g}, 2.53 \mathrm{mmol})$ in $\mathrm{CH}_{2} \mathrm{Cl}_{2}(40 \mathrm{~mL})$ was added $\mathrm{MsCl}(0.48 \mathrm{~mL}, 6.33 \mathrm{mmol})$, followed by the addition of a solution of DMAP (0.61 g, $5.0 \mathrm{mmol})$ in $\mathrm{CH}_{2} \mathrm{Cl}_{2}(10 \mathrm{~mL})$ over $30 \mathrm{~min}$. After the addition was complete additional DMAP $(0.61 \mathrm{~g}, 5.0 \mathrm{mmol})$ in $\mathrm{CH}_{2} \mathrm{Cl}_{2}(10 \mathrm{~mL})$ was added and the reaction mixture was heated on an oil bath to $45^{\circ} \mathrm{C}$ until a clear brown solution was formed $(1 \mathrm{~h})$. The reaction mixture was cooled and diluted with $\mathrm{CH}_{2} \mathrm{Cl}_{2}(200 \mathrm{~mL})$. The organic layer was washed with water $\left(20 \mathrm{~mL}\right.$ ), dried (anhyd $\mathrm{Na}_{2} \mathrm{SO}_{4}$ ), and concentrated to afford a brown oil. Flash chromatography (25\% EtOAc/hexane) gave $0.85 \mathrm{~g}(81 \%)$ of 7 as a clear oil. $\mathrm{R}_{f}=0.6\left(25 \%\right.$ EtOAc/hexane). ${ }^{1} \mathrm{H}$ NMR $\left(400 \mathrm{MHz}, \mathrm{CDCl}_{3}\right) \delta 0.84-1.11(\mathrm{~m}$, $21 \mathrm{H}), 1.77(\mathrm{~m}, 2 \mathrm{H}), 2.24(\mathrm{~m}, 2 \mathrm{H}), 2.40(\mathrm{dd}, J=8.0,20.0 \mathrm{~Hz}, 1 \mathrm{H}) ; 2.55-2.62$ (overlapping dd and $\mathrm{m}, J=6.1,17.8 \mathrm{~Hz}, 2 \mathrm{H}), 2.85(\mathrm{~m}, 1 \mathrm{H}), 3.48(\mathrm{t}, J=6.3 \mathrm{~Hz}, 2 \mathrm{H}), 4.57(\mathrm{~m}, 1 \mathrm{H})$; $4.49(\mathrm{~s}, 2 \mathrm{H}), 6.60(\mathrm{~m}, 1 \mathrm{H}), 7.27-7.36(\mathrm{~m}, 5 \mathrm{H}) ;{ }^{13} \mathrm{C} \mathrm{NMR}\left(100.6 \mathrm{MHz}, \mathrm{CDCl}_{3}\right) \delta$ 12.0, $17.9,26.3,28.4,37.7,49.0,67.8,69.3,72.9,127.5,128.3,136.0,136.4,138.3,204.2$; IR 
(neat) 2906, 1725, $1653 \mathrm{~cm}^{-1}$; MS (CI) $\mathrm{m} / z 417\left(\mathrm{M}^{+}+1\right), 273,243$, 91; HRMS calcd for $\mathrm{C}_{25} \mathrm{H}_{41} \mathrm{O}_{3} \mathrm{Si}\left(\mathrm{M}^{+}+1\right): 417.2825$, found 417.2805; $[\alpha]_{\mathrm{D}}+14.7\left(c 0.58, \mathrm{CHCl}_{3}\right)$.

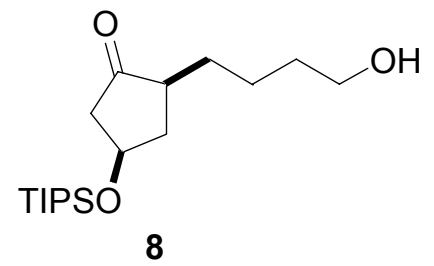

$(3 R, 5 S)-(+)-5-(4$ '-Hydroxybutyl)-3-triisopropylsiloxy-cyclopentanone (8). To the enone $7(1.2 \mathrm{~g}, 2.88 \mathrm{mmol})$ in $\mathrm{EtOH}(15 \mathrm{~mL}), 10 \% \mathrm{Pd} / \mathrm{C}(0.23 \mathrm{~g})$ was added. The reaction mixture was hydrogenated using a $\mathrm{H}_{2}$ balloon for $10 \mathrm{~h}$. After this time the reaction mixture was filtered through a pad of Celite and the pad was washed with additional EtOAc $(50 \mathrm{~mL})$. The organic layer was concentrated and purified by flash chromatography ( $25 \%$ EtOAc/hexane) to afford $900 \mathrm{mg}(95 \%)$ of $\mathbf{8}$ as a clear oil. $\mathrm{R}_{f}=$ $0.2\left(25 \%\right.$ EtOAc/Hexane); ${ }^{1} \mathrm{H}$ NMR $\left(400 \mathrm{MHz}, \mathrm{CDCl}_{3}\right) \delta$ 0.97-1.12 (m, 21H), $1.30(\mathrm{~m}$, 1H), $1.43(\mathrm{~m}, 3 \mathrm{H}), 1.56(\mathrm{~m}, 2 \mathrm{H}), 1.65(\mathrm{~m}, 1 \mathrm{H}), 1.83(\mathrm{~m}, 1 \mathrm{H}), 2.12(\mathrm{~m}, 1 \mathrm{H}), 2.23(\mathrm{dd}, J=$ 6.7, $18.2 \mathrm{~Hz}, 1 \mathrm{H}), 2.41(\mathrm{~m}, 1 \mathrm{H}), 2.59$ (dd, $J=6.5,18.2 \mathrm{~Hz}, 1 \mathrm{H}), 3.64$ (q, $J=8.0 \mathrm{~Hz}, 2 \mathrm{H})$, $4.49(\mathrm{~m}, 1 \mathrm{H}) ;{ }^{13} \mathrm{C} \mathrm{NMR}\left(100.6 \mathrm{MHz}, \mathrm{CDCl}_{3}\right) \delta$ 12.0, 17.9, 23.8, 30.0, 32.5, 39.0, 48.4, 48.6, 62.5, 68.7, 218.3; IR (neat) 3398, 2938, $1738 \mathrm{~cm}^{-1}$; MS (CI) $\mathrm{m} / \mathrm{z} 329\left(\mathrm{M}^{+}+1\right), 155$; HRMS calcd for $\mathrm{C}_{18} \mathrm{H}_{37} \mathrm{O}_{3} \mathrm{Si}\left(\mathrm{M}^{+}+1\right)$ : 329.2512 , found 329.2523; $[\alpha]_{\mathrm{D}}+61.4$ (c 0.23, $\left.\mathrm{CHCl}_{3}\right)$. 


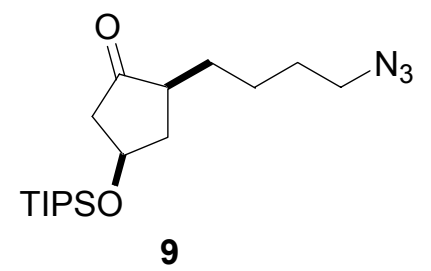

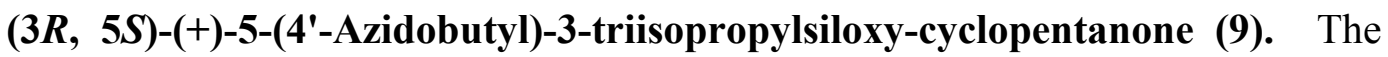
keto alcohol $8(0.549 \mathrm{~g}, 1.67 \mathrm{mmol})$ was dissolved in toluene $(50 \mathrm{~mL})$ and cooled to $0{ }^{\circ} \mathrm{C}$. To the reaction mixture was added $\mathrm{PPh}_{3}(1.32 \mathrm{~g}, 5.0 \mathrm{mmol})$ followed by $\mathrm{Zn}\left(\mathrm{N}_{3}\right)_{2} \cdot 2 \mathrm{pyr}$ (770 mg, $2.5 \mathrm{mmol}$ ). After the reaction mixture was stirred for $15 \mathrm{~min}$ at $0{ }^{\circ} \mathrm{C}$, diisopropyl azodicarboxylate (DIAD) (1.15 g, $5.0 \mathrm{mmol})$ was added carefully over 20 min. The reaction mixture was stirred and allowed to warm up to room temperature over $17 \mathrm{~h}$. After this time $\mathrm{Et}_{2} \mathrm{O}(200 \mathrm{~mL})$ was added, and the organic layer was washed with $\mathrm{H}_{2} \mathrm{O}\left(20 \mathrm{~mL}\right.$ ), dried (anhyd $\mathrm{Na}_{2} \mathrm{SO}_{4}$ ), and concentrated to a pale yellow oil. Flash chromatography (10\% EtOAc/hexane) gave $490 \mathrm{mg}(83 \%)$ of 9 as a pale yellow oil. $\mathrm{R}_{f}=$ $0.8\left(25 \%\right.$ EtOAc/Hexane). ${ }^{1} \mathrm{H}$ NMR $\left(400 \mathrm{MHz}, \mathrm{CDCl}_{3}\right) \delta$ 0.97-1.12 (m, 21H), $1.42(\mathrm{~m}$, 3H), $1.56(\mathrm{~m}, 2 \mathrm{H}), 1.67(\mathrm{~m}, 1 \mathrm{H}), 1.83(\mathrm{~m}, 1 \mathrm{H}), 2.10(\mathrm{~m}, 1 \mathrm{H}), 2.24(\mathrm{dd}, J=6.5,18.2 \mathrm{~Hz}$, 1H), $2.41(\mathrm{~m}, 1 \mathrm{H}), 2.59(\mathrm{dd}, J=6.5,18.2 \mathrm{~Hz}, 1 \mathrm{H}), 3.27(\mathrm{t}, J=6.8 \mathrm{~Hz}, 2 \mathrm{H}), 4.49(\mathrm{~m}, 1 \mathrm{H})$; ${ }^{13} \mathrm{C}$ NMR $\left(100.6 \mathrm{MHz}, \mathrm{CDCl}_{3}\right) \delta 11.9,17.8,24.7,28.7,29.8,38.9,48.5,48.1,51.1,68.7$, 217.9; IR (neat) 2917, 2095, $1743 \mathrm{~cm}^{-1}$; MS (CI) $\mathrm{m} / z 354\left(\mathrm{M}^{+}+1\right), 326,282,152$; HRMS calcd for $\mathrm{C}_{18} \mathrm{H}_{36} \mathrm{~N}_{3} \mathrm{O}_{2} \mathrm{Si}\left(\mathrm{M}^{+}+1\right)$ : 354.2577, found 354.2552; $[\alpha]_{\mathrm{D}}+51.9$ (c 0.29, $\left.\mathrm{CHCl}_{3}\right)$. 


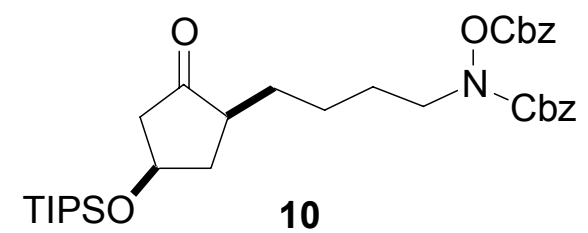

(2S,4R)-(+)-4-Triisopropylsiloxy-2-(4'-N,O-di(benzyloxycarbamyl)hydroxylaminobutyl)cyclopetan-1-one (10). The ketone alcohol $8(320 \mathrm{mg}, 0.97 \mathrm{mmol})$ was dissolved in THF $(15 \mathrm{~mL})$ and cooled in an ice-water bath under argon. To this reaction mixture was added $N, O$-di(benzyloxycarbamyl)hydroxylamine (293 $\mathrm{mg}, 0.97 \mathrm{mmol}$ ), followed by the addition of $\mathrm{PPh}_{3}(417 \mathrm{mg}, 1.5 \mathrm{mmol})$. The resulting mixture was stirred in ice-water bath for $15 \mathrm{~min}$. After this time the DIPAD (344 mg, $1.5 \mathrm{mmol}$ ) in $2 \mathrm{~mL}$ THF was added via a syringe pump over 40 min. After addition, the mixture was stirred for another $1.5 \mathrm{~h}$ in ice-water bath. Concentration followed by flash chromatography (2$10 \% \mathrm{EtOAc} /$ hexane $)$ afforded $530 \mathrm{mg}(90 \%)$ of compound $\mathbf{1 0}$ as an oil. $\mathrm{R}_{f}=0.42(5: 1$ hexane/EtOAc). ${ }^{1} \mathrm{H}$ NMR (400 MHz, $\left.\mathrm{CDCl}_{3}\right) \delta 1.08(\mathrm{~m}, 21 \mathrm{H}), 1.40(\mathrm{~m}, 3 \mathrm{H}), 1.62(\mathrm{~m}$, 3H), $1.82(\mathrm{~m}, 1 \mathrm{H}), 2.10(\mathrm{~m}, 1 \mathrm{H}), 2.24(\mathrm{dd}, J=6.9,18.3 \mathrm{~Hz}, 1 \mathrm{H}), 2.41(\mathrm{~m}, 1 \mathrm{H}), 2.57(\mathrm{dd}$, $J=6.5,18.2 \mathrm{~Hz}, 1 \mathrm{H}), 3.68(\mathrm{t}, J=6.9 \mathrm{~Hz}, 1 \mathrm{H}), 4.49(\mathrm{~m}, 1 \mathrm{H}), 5.20(\mathrm{~s}, 2 \mathrm{H}), 5.26(\mathrm{~s}, 2 \mathrm{H})$, $7.36(\mathrm{~m}, 10 \mathrm{H}) ;{ }^{13} \mathrm{C} \mathrm{NMR}\left(100.6 \mathrm{MHz}, \mathrm{CDCl}_{3}\right) \delta 12.4,18.4,24.9,27.2,30.3,39.4,48.8$, $49.0,50.9,68.7,69.2,71.6,128.3,128.7,128.8,128.9,129.1,129.3,134.6,135.9,154.7$, 156.1, 218.3; IR (neat) 1791, $1740 \mathrm{~cm}^{-1}$; MS (ESI) $\mathrm{m} / z 612\left(\mathrm{M}^{+}+1\right)$; HRMS calcd for $\mathrm{C}_{34} \mathrm{H}_{53} \mathrm{~N}_{2} \mathrm{O}_{7} \mathrm{Si}\left(\mathrm{M}^{+}+\mathrm{NH}_{4}{ }^{+}\right): 629.3622$, found 629.3646. $[\alpha]_{\mathrm{D}}+32.02\left(\mathrm{c} 1.03, \mathrm{CHCl}_{3}\right)$. 


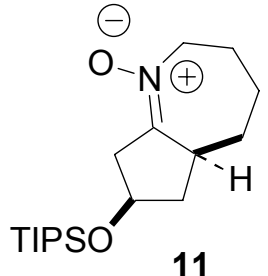

(5aS,7R)-(-)-7-Triisopropylsiloxy-2,3,4,5,5a,6,7,8-octahydrocyclopent [b]-

azepine-N-oxide (11). Compound 10 (250 $\mathrm{mg}, 0.41 \mathrm{mmol})$ was dissolved in EtOH (3.5 $\mathrm{mL}$ ) and $10 \% \mathrm{Pd}-\mathrm{C}(28 \mathrm{mg})$ was added. The resulting mixture was hydrogenated at atmosphere (balloon) for $6 \mathrm{~h}$. Concentration followed by chromatography (10\% EtOAc/hexane, followed by $1-2 \% \mathrm{MeOH} / \mathrm{CHCl}_{3}$ ) gave $120 \mathrm{mg}(90 \%)$ of nitrone $\mathbf{1 1}$ as an oil. $\mathrm{R}_{f}=0.28\left(20: 1 \mathrm{MeOH} / \mathrm{CHCl}_{3}\right) .{ }^{1} \mathrm{H}$ NMR $\left(400 \mathrm{MHz}, \mathrm{CDCl}_{3}\right) \delta 1.05(\mathrm{~m}, 21 \mathrm{H}), 1.57-$ 2.31 (complex, 8H), $2.72(\mathrm{~m}, 2 \mathrm{H}), 2.99(\mathrm{dd}, J=5.5,19.6 \mathrm{~Hz}, 1 \mathrm{H}), 3.99-4.30(\mathrm{~m}, 2 \mathrm{H})$, $4.38(\mathrm{~m}, 1 \mathrm{H}) ;{ }^{13} \mathrm{C}$ NMR $\left(100.6 \mathrm{MHz}, \mathrm{CDCl}_{3}\right) \delta 12.4,18.4,25.9,30.0,33.0,41.4,41.9$, 44.0, 66.7, 71.0, 156.3; IR (neat) $1636 \mathrm{~cm}^{-1}$; MS (EI) $\mathrm{m} / z 326\left(\mathrm{M}^{+}+1\right)$; HRMS calcd for $\mathrm{C}_{18} \mathrm{H}_{36} \mathrm{NO}_{2} \mathrm{Si}\left(\mathrm{M}^{+}+1\right)$ : 326.2515 , found 326.2514. $[\alpha]_{\mathrm{D}} \quad-50.75$ (c 1.30, $\left.\mathrm{CHCl}_{3}\right)$. This nitrone was used immediately in the next step.

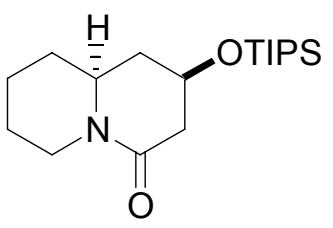

$3 a$

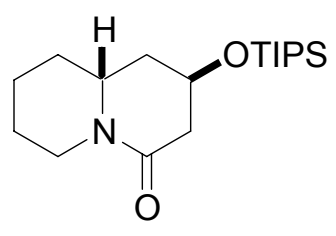

3b

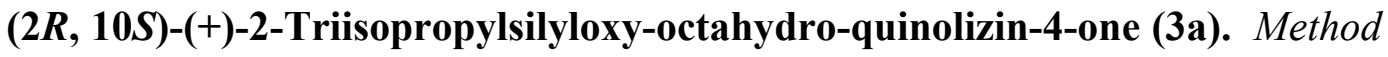
A. To the keto azide 9 (300 $\mathrm{mg}, 0.85 \mathrm{mmol})$ in $\mathrm{CH}_{2} \mathrm{Cl}_{2}(15 \mathrm{~mL})$ at $0{ }^{\circ} \mathrm{C}$ was added freshly distilled $\mathrm{TiCl}_{4}(0.23 \mathrm{~mL}, 2.12 \mathrm{mmol})$ over $5 \mathrm{~min}$. The reaction mixture was 
allowed to warm to room temperature over $20 \mathrm{~min}$, and then heated to $45^{\circ} \mathrm{C}$ for $11 \mathrm{~h}$. After cooling, $5 \mathrm{~mL} 15 \%$ aqueous $\mathrm{NaOH}$ was carefully added and the mixture stirred for an additional $30 \mathrm{~min}$. The pale yellow solution was extracted into $\mathrm{CH}_{2} \mathrm{Cl}_{2}(300 \mathrm{~mL})$, dried (anhyd $\mathrm{MgSO}_{4}$ ), and concentrated to a pale yellow oil. Chromatography (25\% EtOAc/hexane) gave $218 \mathrm{mg}(79 \%)$ of lactams $\mathbf{3 a}$ and $\mathbf{3 b}$ as a (1:1.3) mixture of diastereoisomers. A second chromatographic separation (10\% EtOAc/hexane) afforded analytical samples of $\mathbf{3 a}$ and 3b. For lactam 3a: $\mathrm{R}_{f}=0.73\left(50 \%\right.$ EtOAc/hexane). ${ }^{1} \mathrm{H}$ NMR $\left(400 \mathrm{MHz}, \mathrm{CDCl}_{3}\right) \delta 1.04-1.10(\mathrm{~m}, 21 \mathrm{H}), 1.26-1.83(\mathrm{~m}, 7 \mathrm{H}), 2.16(\mathrm{~m}, 1 \mathrm{H}), 2.32-$ $2.40(\mathrm{~m}, 2 \mathrm{H}), 2.72(\mathrm{ddd}, J=2.8,7.6,16.5 \mathrm{~Hz}, 1 \mathrm{H}), 3.18(\mathrm{~m}, 1 \mathrm{H}), 4.01(\mathrm{~m}, 1 \mathrm{H}), 4.71-4.75$ $(\mathrm{m}, 1 \mathrm{H}) ;{ }^{13} \mathrm{C} \mathrm{NMR}\left(100.6 \mathrm{MHz}, \mathrm{CDCl}_{3}\right) \delta 12.6,18.4,24.8,25.6,34.9,40.9,42.5,43.4$, 54.8, 65.0, 168.7; IR (neat) 2941, 2865, $1650 \mathrm{~cm}^{-1}$; MS (CI) $m / z 326\left(\mathrm{M}^{+}+1\right), 282$; HRMS calcd for $\mathrm{C}_{18} \mathrm{H}_{36} \mathrm{NO}_{2} \mathrm{Si}\left(\mathrm{M}^{+}+1\right)$ : 326.2515 , found $326.2534 ;[\alpha]_{\mathrm{D}}+14.2^{\circ}(\mathrm{c} 0.13$, $\left.\mathrm{CHCl}_{3}\right)$. For lactam 3b: $\mathrm{R}_{f}=0.71\left(50 \%\right.$ EtOAc/hexane). ${ }^{1} \mathrm{H}$ NMR $\left(400 \mathrm{MHz}, \mathrm{CDCl}_{3}\right) \delta$ 0.99-1.05 (m, 21H), $1.27(\mathrm{~m}, 1 \mathrm{H}), 1.42(\mathrm{~m}, 2 \mathrm{H}), 1.63(\mathrm{~m}, 1 \mathrm{H}), 1.70(\mathrm{~m}, 2 \mathrm{H}), 1.80(\mathrm{~m}$, 1H), $1.98(\mathrm{~m}, 1 \mathrm{H}), 2.38-2.54(\mathrm{~m}, 3 \mathrm{H}), 3.34(\mathrm{~m}, 1 \mathrm{H}), 4.26(\mathrm{~m}, 1 \mathrm{H}), 4.73(\mathrm{~m}, 1 \mathrm{H}) ;{ }^{13} \mathrm{C}$ NMR $\left(100.6 \mathrm{MHz}, \mathrm{CDCl}_{3}\right) \delta 12.1,17.9,24.5,25.3,33.9,38.3,41.7,42.0,52.8,63.8$, 167.8; IR (neat) 2933, 2862, $1648 \mathrm{~cm}^{-1}$; MS (CI) $m / z 326\left(\mathrm{M}^{+}+1\right), 282$; HRMS calcd for $\mathrm{C}_{18} \mathrm{H}_{36} \mathrm{NO}_{2} \mathrm{Si}\left(\mathrm{M}^{+}+1\right): 326.2515$, found 326.2508; $[\alpha]_{\mathrm{D}}-9.5\left(\mathrm{c} 0.09, \mathrm{CHCl}_{3}\right)$.

Method B. A degassed mixture of nitrone 11 (60 $\mathrm{mg}, 0.5 \mathrm{mmol})$ in $\mathrm{CH}_{3} \mathrm{CN} /$ toluene $(4: 1,20 \mathrm{~mL})$ was photolyzed in a capped quartz tube $(254 \mathrm{~nm}$, MerryGo-Round apparatus) for $2 \mathrm{~h}$. Concentration followed by chromatography $(10 \%$ 
EtOAc/hexane, followed by $\left.1-2 \% \mathrm{MeOH} / \mathrm{CHCl}_{3}\right)$ afforded $36 \mathrm{mg}(60 \%)$ of $\mathbf{3 a}$ isolated as an oil.

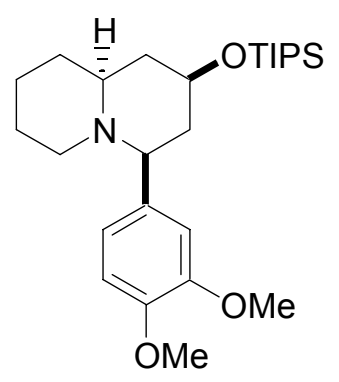

12

\section{4-(1-Aza-4-triisopropylsilyloxy-bicyclo[4.4.0]dec-2-yl-1,2-dimethoxybenzene}

(12). To a dry flask containing anhydrous $\mathrm{CeCl}_{3}(182 \mathrm{mg}, 0.740 \mathrm{mmoL})$ was added a solution of lactam 3a (60 mg, $0.185 \mathrm{mmoL})$ in $2 \mathrm{~mL}$ dry THF. After stirring for an hour at room temperature, the reaction mixture was cooled to $0{ }^{\circ} \mathrm{C}$, and a solution of Grignard reagent of 4-bromoveratrole in THF $(1.5 \mathrm{~mL}, 1.48 \mathrm{mmoL})$ was added dropwise. The mixture was allowed to warm to room temperature and stirred overnight. It was then cooled to $0{ }^{\circ} \mathrm{C}$ and $\mathrm{NaBH}_{3} \mathrm{CN}(70 \mathrm{mg}, 1.11 \mathrm{mmoL})$ followed by glacial acetic acid $(0.25$ $\mathrm{mL})$ were added. After stirring for an hour, $\mathrm{Et}_{2} \mathrm{O}(50 \mathrm{~mL})$ was added and the mixture was washed with $15 \%$ aq $\mathrm{KOH}(2 \times 5 \mathrm{~mL})$. The organic layer was dried $\left(\mathrm{Na}_{2} \mathrm{SO}_{4}\right)$, concentrated and purified by chromatography ( $10 \%$ ethyl acetate/hexanes) to afford 70 mg $(85 \%)$ of 12 as a clear oil. ${ }^{1} \mathrm{H}$ NMR $\left(400 \mathrm{MHz}, \mathrm{CDCl}_{3}\right) \delta 1.01(\mathrm{~m}, 21 \mathrm{H}), 1.21(\mathrm{~m}$, 2H), 1.36-1.69 (m, 7H), 1.83-1.96 (m, 3H), $2.64(\mathrm{~m}, 1 \mathrm{H}), 2.86(\mathrm{dd}, J=4.0,12.0 \mathrm{~Hz}, 1 \mathrm{H})$, $3.77(\mathrm{~m}, 1 \mathrm{H}), 3.86(\mathrm{~s}, 3 \mathrm{H}), 3.89(\mathrm{~s}, 3 \mathrm{H}), 6.75-6.80(\mathrm{~m}, 3 \mathrm{H}) ;{ }^{13} \mathrm{C}$ NMR (100.6 MHz, $\left.\mathrm{CDCl}_{3}\right) \delta 12.4,18.1,24.7,26.2,33.8,43.6,45.8,53.1,55.9,56.0,61.0,68.4,69.1,110.4$ 110.5, 119.6, 119.7, 137.2, 147.8; IR (neat) 2935, 2863, 2786, $2747 \mathrm{~cm}^{-1}$; MS (CI) $\mathrm{m} / z$ 
$448\left(\mathrm{M}^{+}+1\right)$, 282; HRMS calcd for $\mathrm{C}_{26} \mathrm{H}_{46} \mathrm{NO}_{3} \mathrm{Si}\left(\mathrm{M}^{+}+1\right)$ : 448.3247, found 448.3263; $[\alpha]_{\mathrm{D}}-48.6\left(\mathrm{c} 0.15, \mathrm{CHCl}_{3}\right)$.

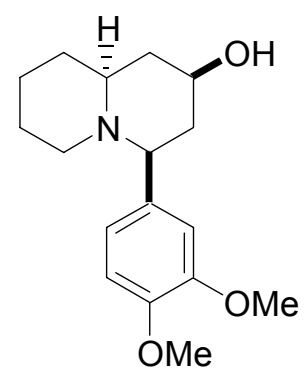

13

2-epi-Lasubine II (13). To the amine $12(53 \mathrm{mg}, 0.118 \mathrm{mmoL})$ in $5 \mathrm{~mL}$ dry THF was added $1.0 \mathrm{M}$ TBAF in THF $(0.12 \mathrm{~mL}, 0.118 \mathrm{mmoL})$ at $0{ }^{\circ} \mathrm{C}$. The mixture was allowed to warm to room temperature and stirred for 3 hours. Concentration and chromatography $\left(10 \% \mathrm{MeOH} / \mathrm{CH}_{2} \mathrm{Cl}_{2}\right)$ afforded $30 \mathrm{mg}$ of $\mathbf{1 3}(87 \%)$ as a clear oil. ${ }^{1} \mathrm{H}$ NMR (400 MHz, $\left.\mathrm{CDCl}_{3}\right) \delta$ 1.24-2.02 (complex, 14H), $2.64(\mathrm{~m}, 1 \mathrm{H}), 2.84(\mathrm{dd}, J=4.0$, $12.0 \mathrm{~Hz}, 1 \mathrm{H}), 3.73(\mathrm{~m}, 1 \mathrm{H}), 3.86(\mathrm{~s}, 3 \mathrm{H}), 3.89(\mathrm{~s}, 3 \mathrm{H}), 6.75-6.80(\mathrm{~m}, 3 \mathrm{H}) .{ }^{13} \mathrm{C}$ NMR $\left(100.6 \mathrm{MHz}, 50{ }^{\circ} \mathrm{C}, \mathrm{CDCl}_{3}\right) \delta 24.6,26.1,33.8,42.9,45.4,52.8,55.8,55.9,60.8,68.0$, 68.4, 110.7, 111.3, 119.6, 137.1, 148.1, 149.3; IR (neat) 3366, 2934, 2856, $2792 \mathrm{~cm}^{-1}$; MS (CI) m/z $292\left(\mathrm{M}^{+}+1\right), 164,154,126,96,84$; HRMS calcd for $\mathrm{C}_{17} \mathrm{H}_{26} \mathrm{NO}_{3}\left(\mathrm{M}^{+}+1\right)$ : 292.1913, found: 292.1934. $[\alpha]_{\mathrm{D}}-65.5\left(c\right.$ 1.0, $\left.\mathrm{CHCl}_{3}\right)$; $[\alpha]_{\mathrm{D}}-56.9$ (c 1.0, MeOH). Lit. $[\alpha]_{\mathrm{D}}-44.4(c 2.1, \mathrm{MeOH}){ }^{9}$ 


\section{References:}

(1) Deardorff, D. R.; Myles, D. C. Org. Synth. 1989, 67, 114-120.

(2) Johnson, C. R.; Bis, S. J. Tetrahedron Lett. 1992, 33, 7287-90.

(3) Deardorff, D. R.; Windham, C. Q.; Craney, C. L. Org. Synth. 1996, 73, 25-35.

(4) Busato, S.; Tinembart, O.; Zhang, Z. D.; Scheffold, R. Tetrahedron 1990, 46, 3155-3166.

(5) Paquette, L. A.; Heidelbaugh, T. M. Org. Synth. 1996, 73, 44-49.

(6) Johnson, C. R.; Raheja, R. K. J. Org. Chem. 1994, 59, 2287-2288.

(7) Chow, K.; Danishefsky, S. J. J. Org. Chem. 1989, 54, 6016-6018.

(8) Hashimoto, M.; Kan, T.; Yanagiya, M.; Shirahama, H.; Matsumoto, T. Tetrahedron Lett. 1987, 28, 5665-5668.

(9) Ma, D.; Zhu, W. Org. Lett. 2001, 3, 3927-3929. 
S15

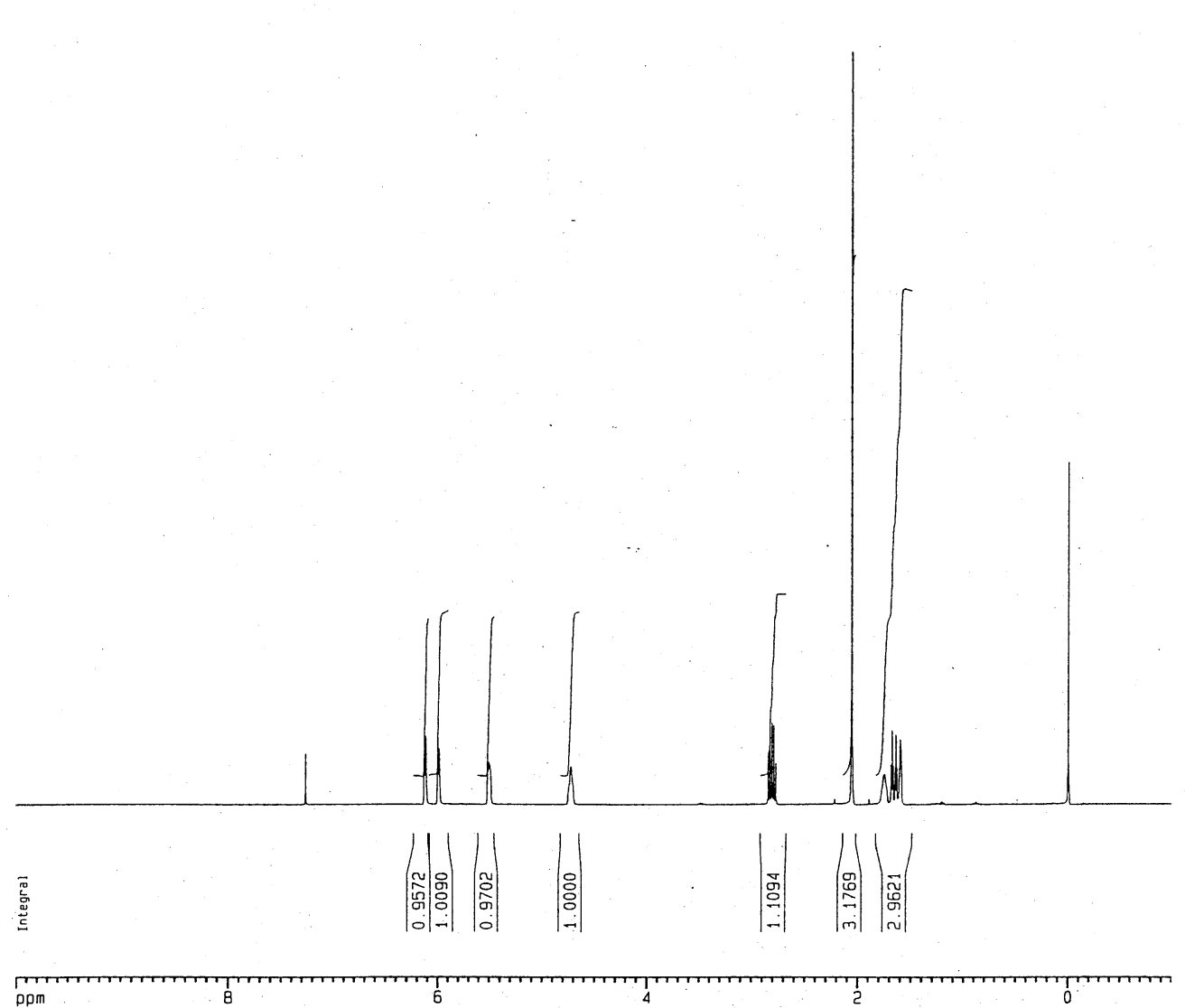

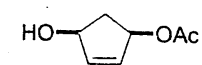

A

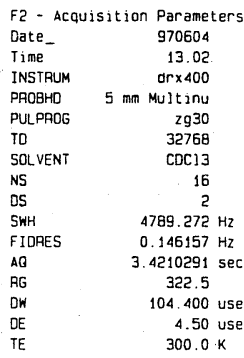

F2 - Processing parameters

$\begin{array}{lc}\mathrm{SI} & 16384 \\ \mathrm{SF} & 400.1300095 \mathrm{MHz}\end{array}$

WSB

SSB
GB

PC

10 NMF plot par

$20.00 \mathrm{~cm}$

FIP $\quad 10.000$ pD

$\begin{array}{ll}F 1 & 4001.30 \mathrm{~Hz} \\ \text { F2P } & -1.000 \mathrm{ppm}\end{array}$

F2 $\quad-400.13 \mathrm{~Hz}$

PPMCM $\quad 0.55000 \mathrm{ppm} / \mathrm{cm}$
票命

兽

言

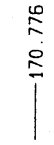

)

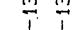

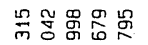

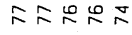

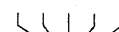

$\forall$
NAME

EXPNC
PROCA

F2 - Acquisition Parameters

Date

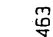

$\stackrel{\circ}{0}$

藏
Iime

INSTRUM

PULPRO

SOLVENT

NS

DS
SWH
FIDRE

FIO

AG

OW
OE
TE

13.46
$05 \times 400$

290930

$29 p 930$
65536

$\operatorname{coc} 13$

1024
2

23148.148 Hz

$0.353213 \mathrm{~Hz}$

$1.4156276 \mathrm{Sec}$

4096

21.600 usec

$300.0 \mathrm{~K}$

$0.0300000 \mathrm{sec}$

$0.0000200 \mathrm{sec}$ 18.00 d8

$0.05000000 \mathrm{sec}$

waltz16

100.00 usec

$400.1316005 \mathrm{MHz}$

$1 \mathrm{H}$

$-6.00 \mathrm{~d}$
$18.00 \mathrm{d \theta}$

$18.00 \mathrm{~dB}$
$6.90 \mathrm{usec}$

4.50 usec

100. $6232933 \mathrm{MHz}$

$13 \mathrm{C}$
$-6.00 \mathrm{~dB}$

F2 - Processing parameters

32768
SI

SF
NOW
100.6127734
EM MHZ

WOW

SSB
LB
GB
PC

10 NMP plot parameters

$\begin{array}{lr}F_{1 P} & 220.000 \mathrm{ppm} \\ \mathrm{F}_{1} & 22134.81 \mathrm{~Hz}\end{array}$

F2P $\quad-10.000 \mathrm{pgm}$

$\begin{array}{ll}\text { F2 } & -1006.13 \mathrm{~Hz}\end{array}$

PPMCM $\quad 11.50000 \mathrm{ppm} / \mathrm{Cm}$ 

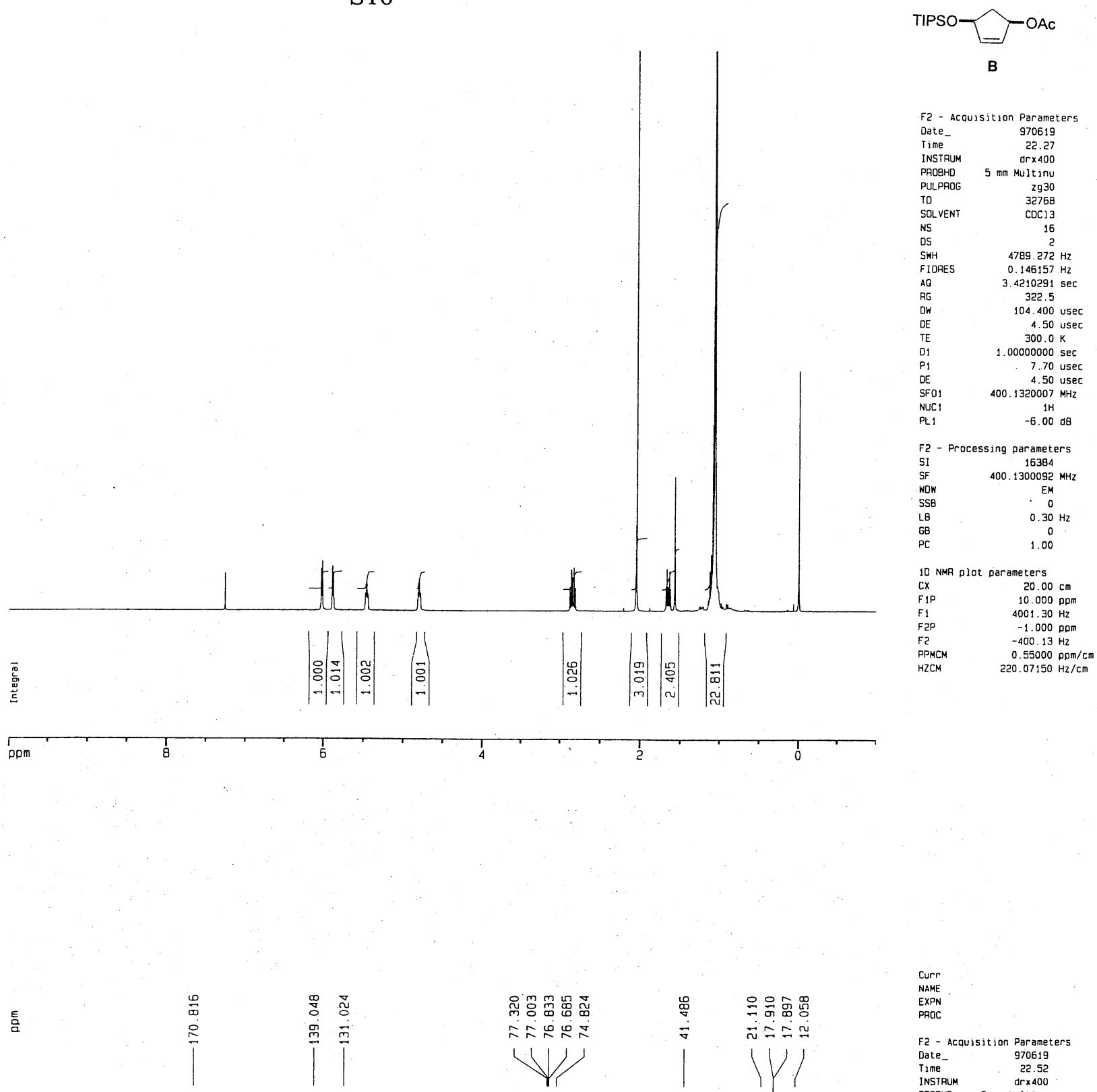

Curr
NAME
EXPN

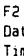

F2 -
Date
Time

PAOBHO

PULPROG

TD

SQL VENT
NS
DS

DS

SWH $23148.148 \mathrm{~Hz}$

FIDRES $\quad 0.353213 \mathrm{~Hz}$

$\begin{array}{lc}\text { AO } & 1.4156276 \mathrm{sec} \\ \text { AG } & 4096\end{array}$

PG

$\mathrm{DE}$
$\mathrm{TE}$
$\mathrm{d}, \mathrm{s}$

21.600 usec

4.50 usec 0.0300000

$18.00 \mathrm{dg}$
$5000000 \mathrm{sec}$ waltz16 100.00 usec

PL 13

CPDPAG2

SFO2

NUC2

PL2
PL12

400. $1316005 \mathrm{MHZ}$

$18.00 \mathrm{dg}$
6.90 usec

$100.6232933 \mathrm{MHz}$

$6232933 \mathrm{MHz}_{13 \mathrm{C}}$

$\begin{array}{ll}\text { NUC1 } & 13 \mathrm{C} \\ \text { PL1 } & -6.00 \mathrm{~d}\end{array}$

$\mathrm{FZ}$ - Processing parameter
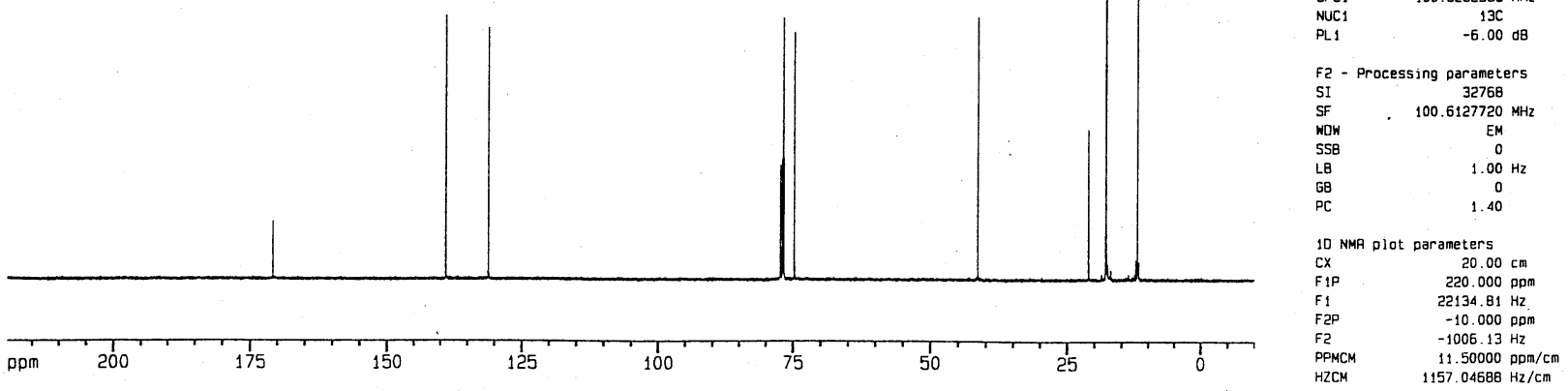

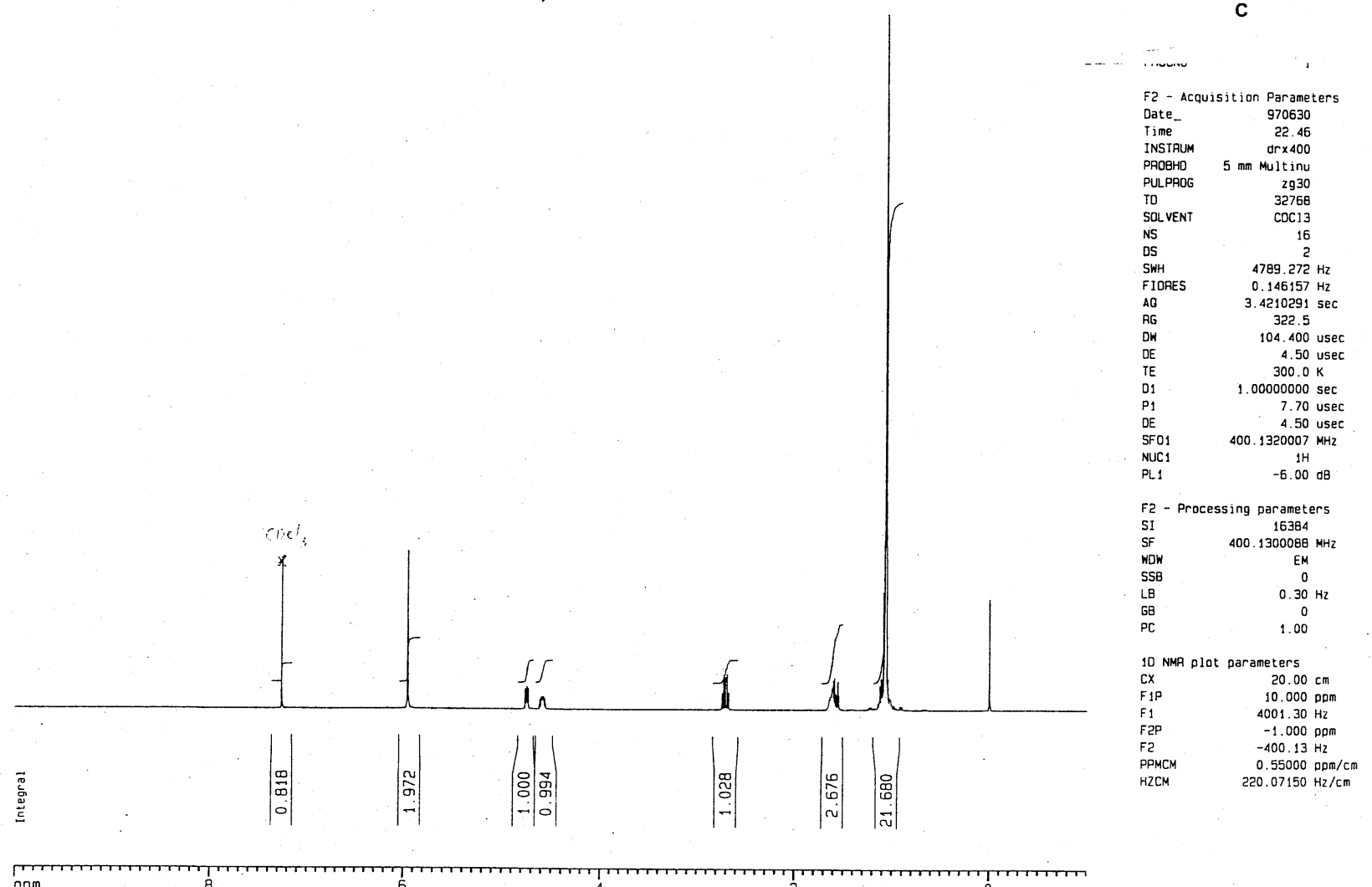

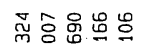

却是员

Wl
㟔塄

$\approx \cong$

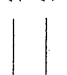

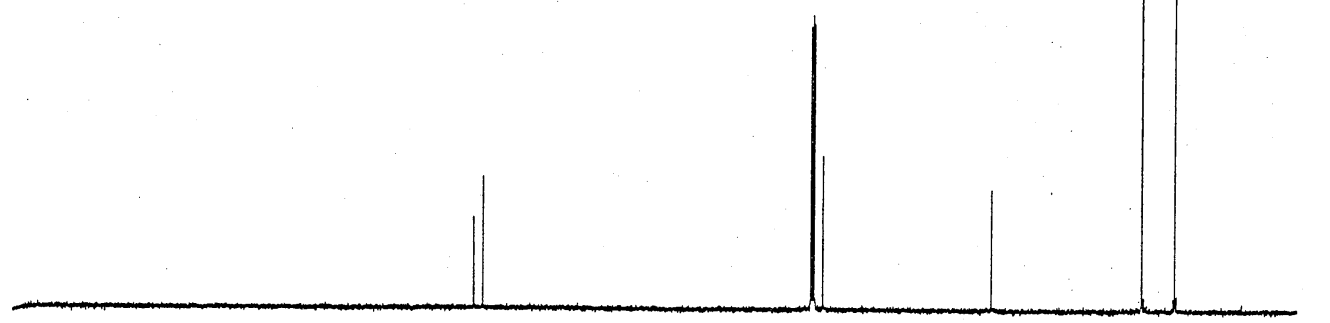

$$
\text { ppm }
$$
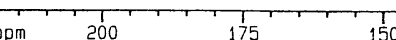

150

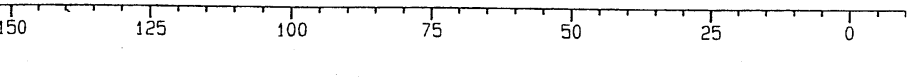

100
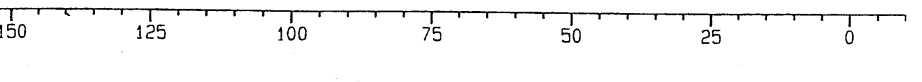


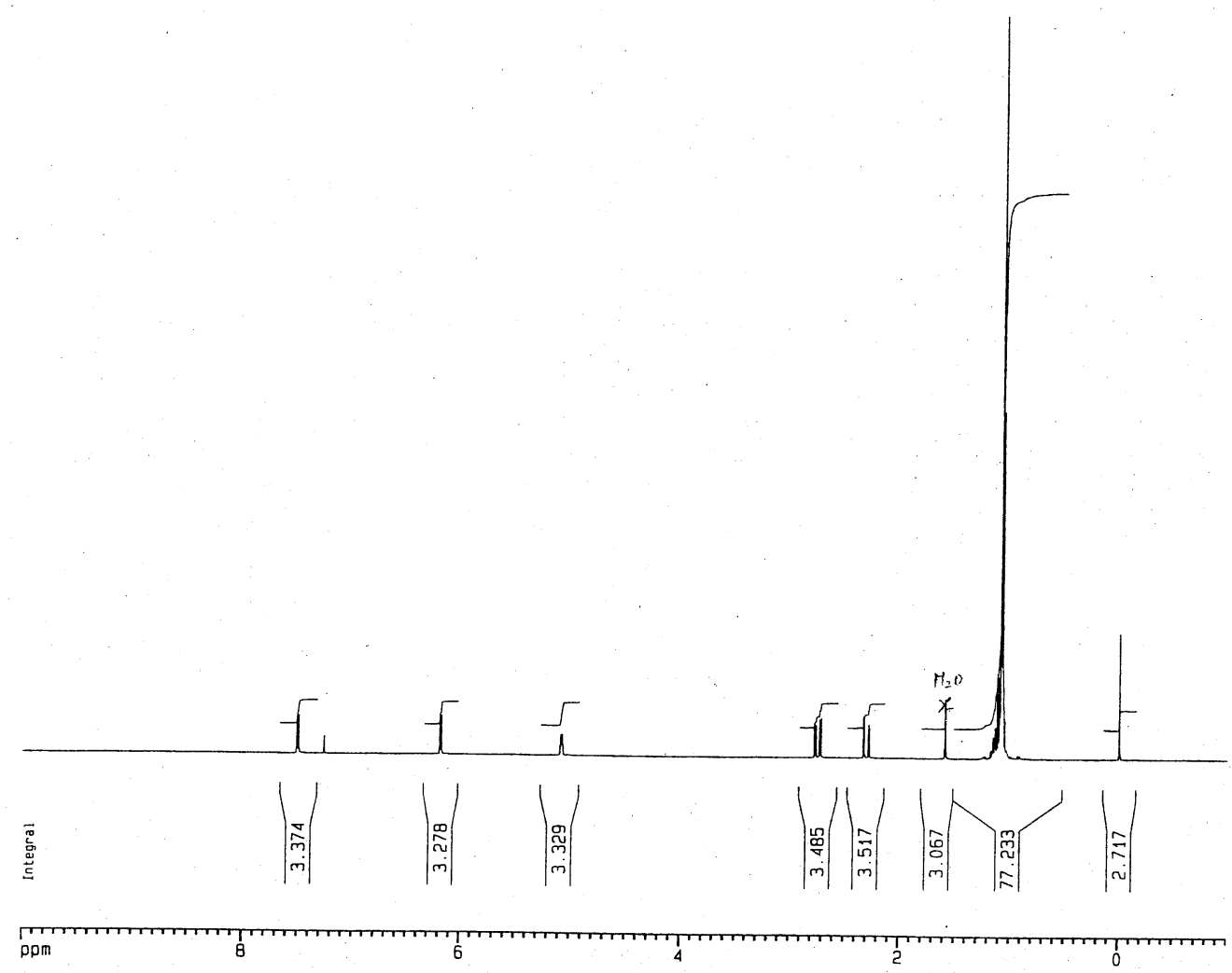

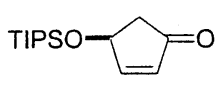
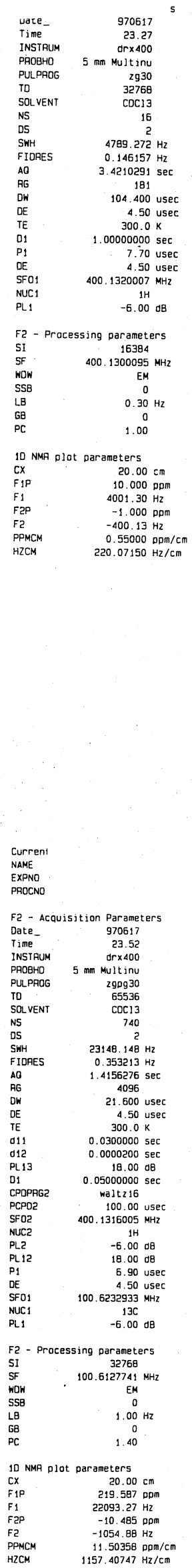

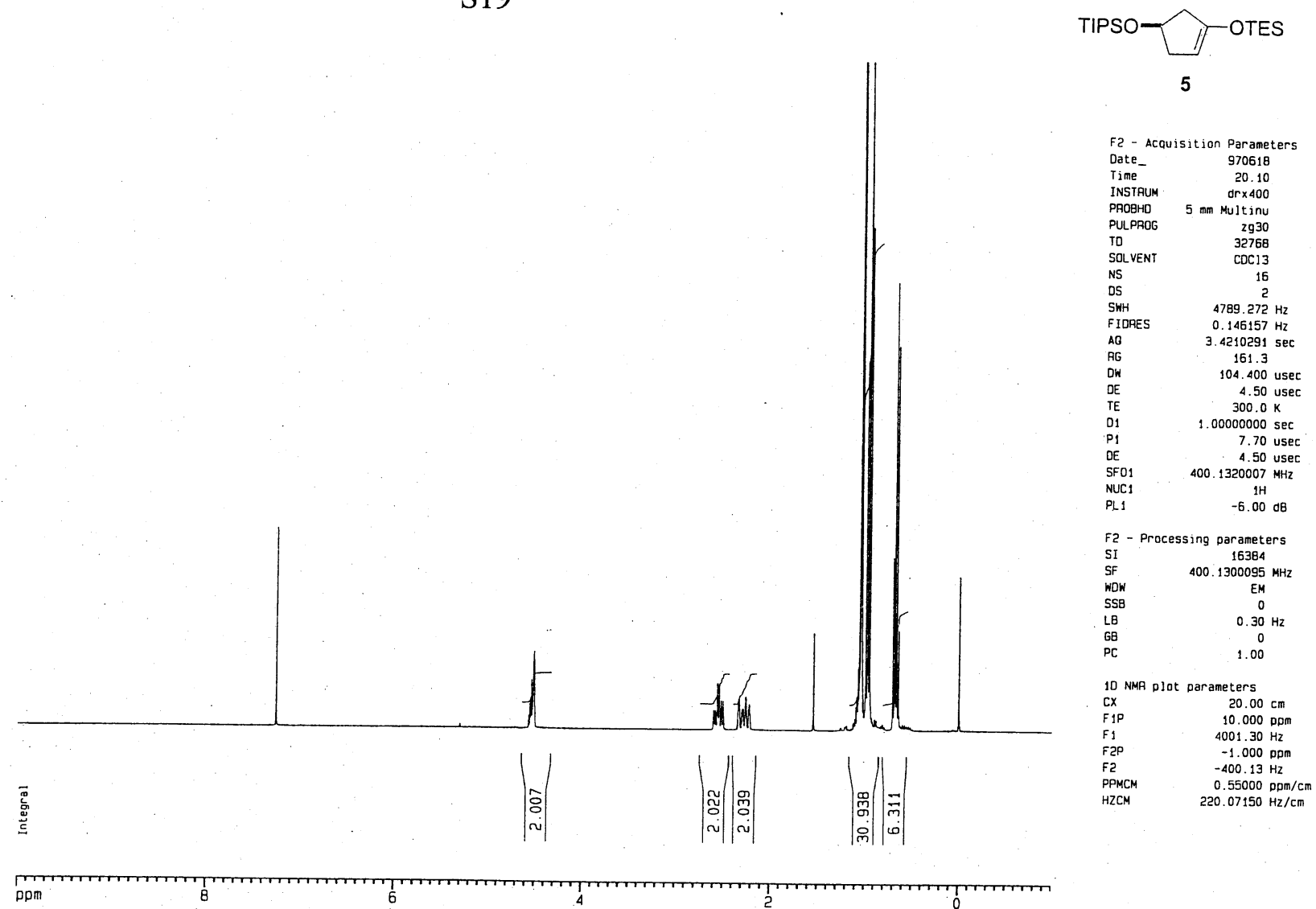

틈
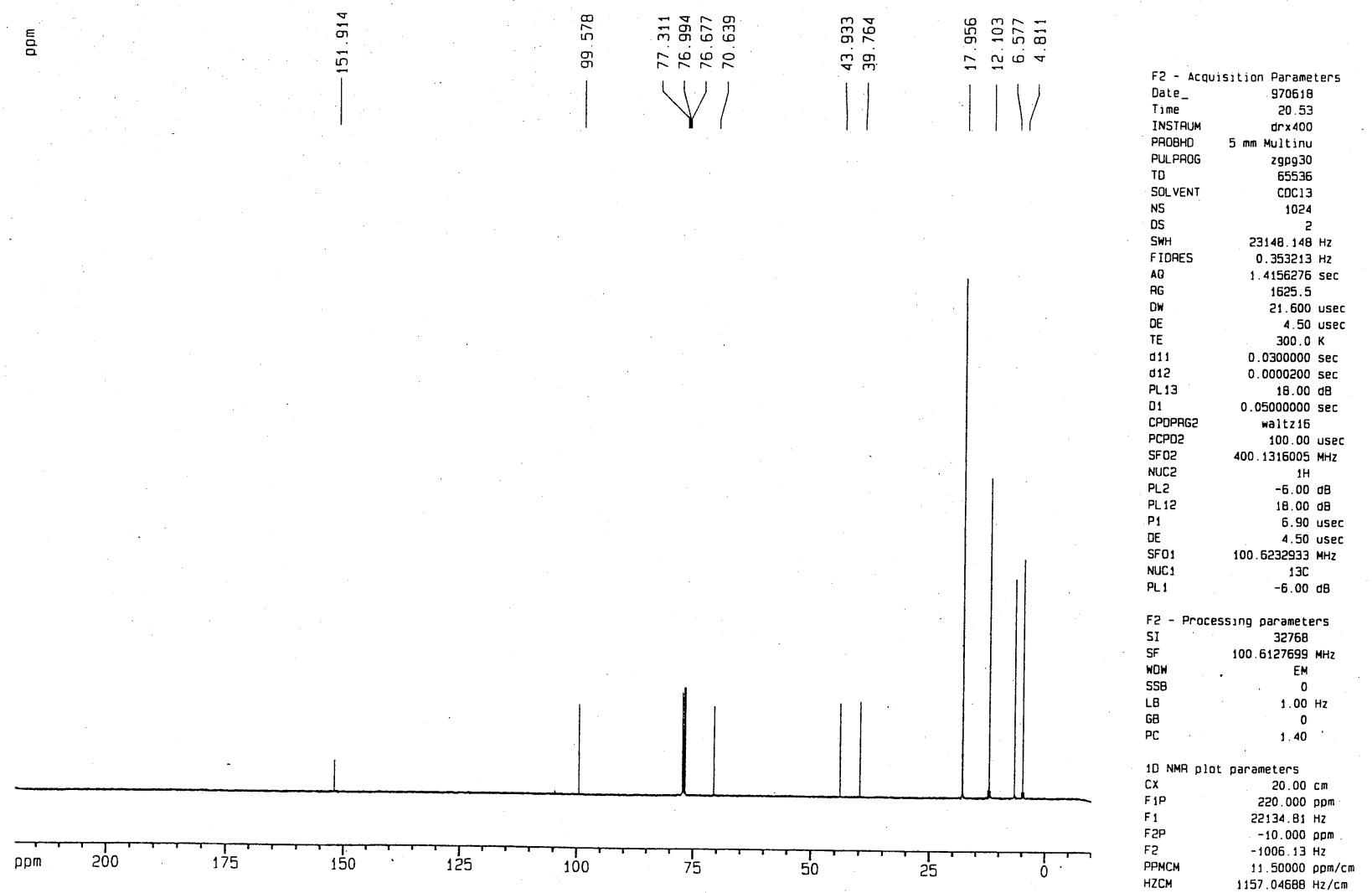

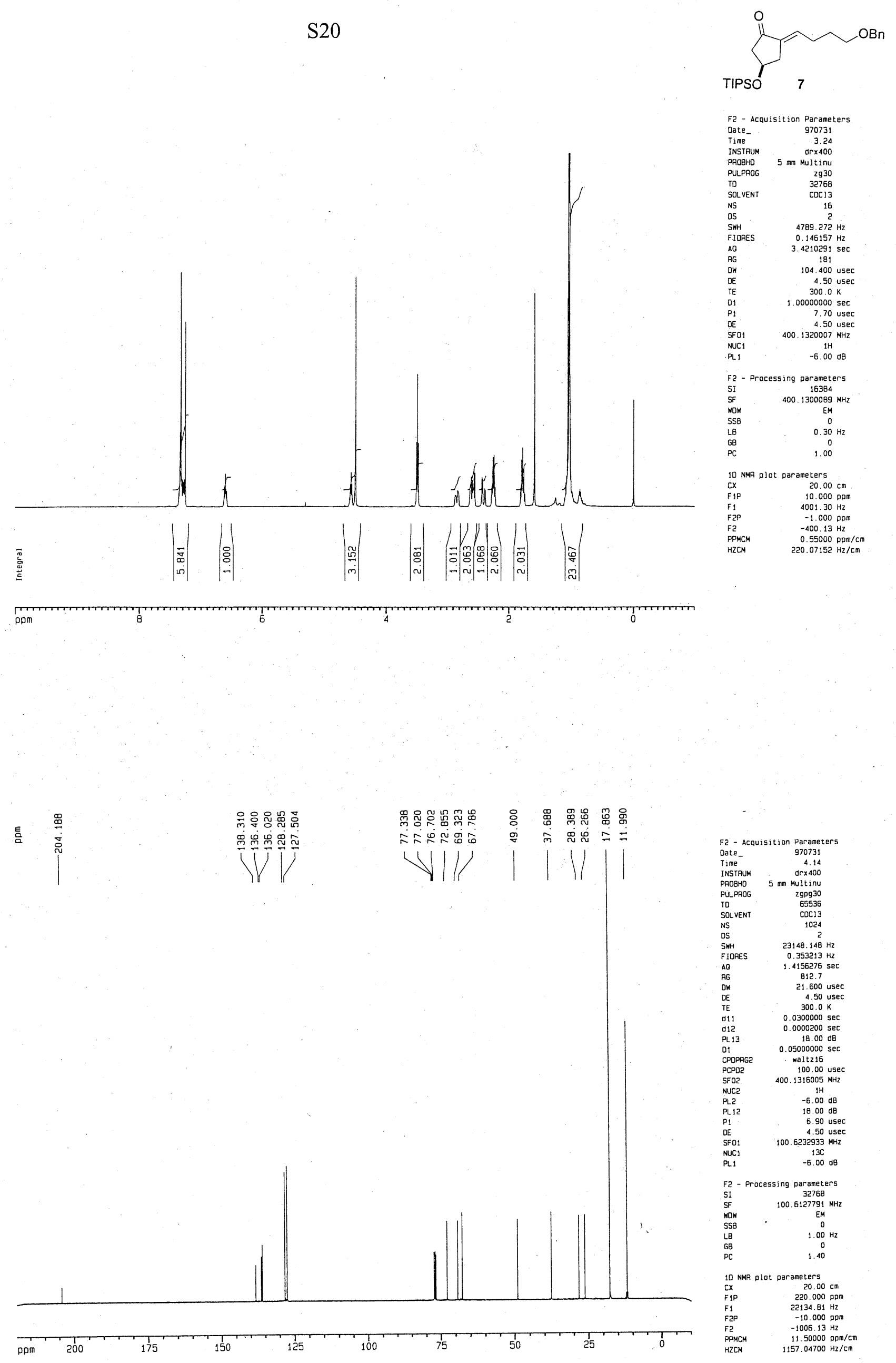

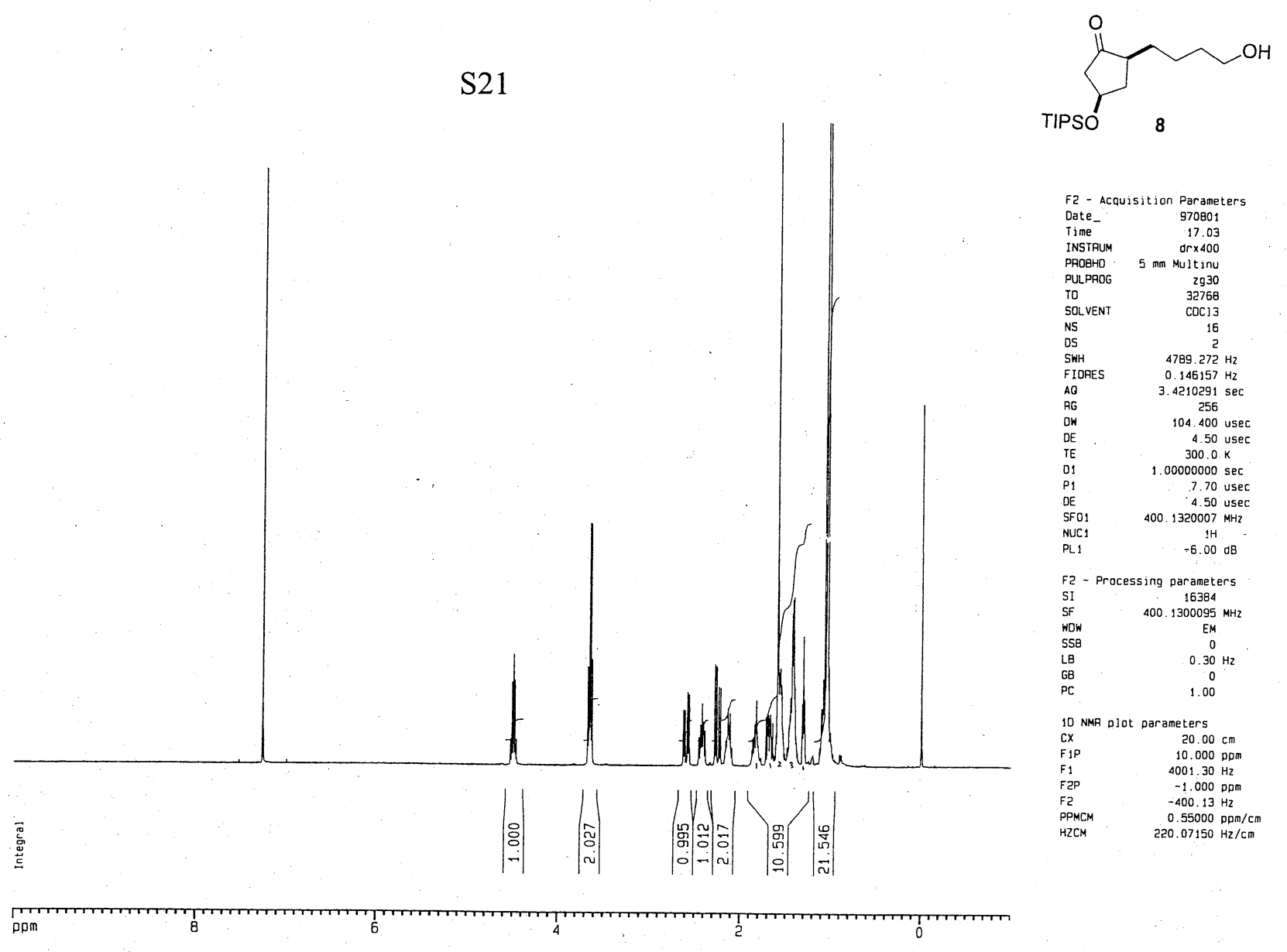

ja

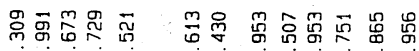

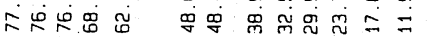

vil
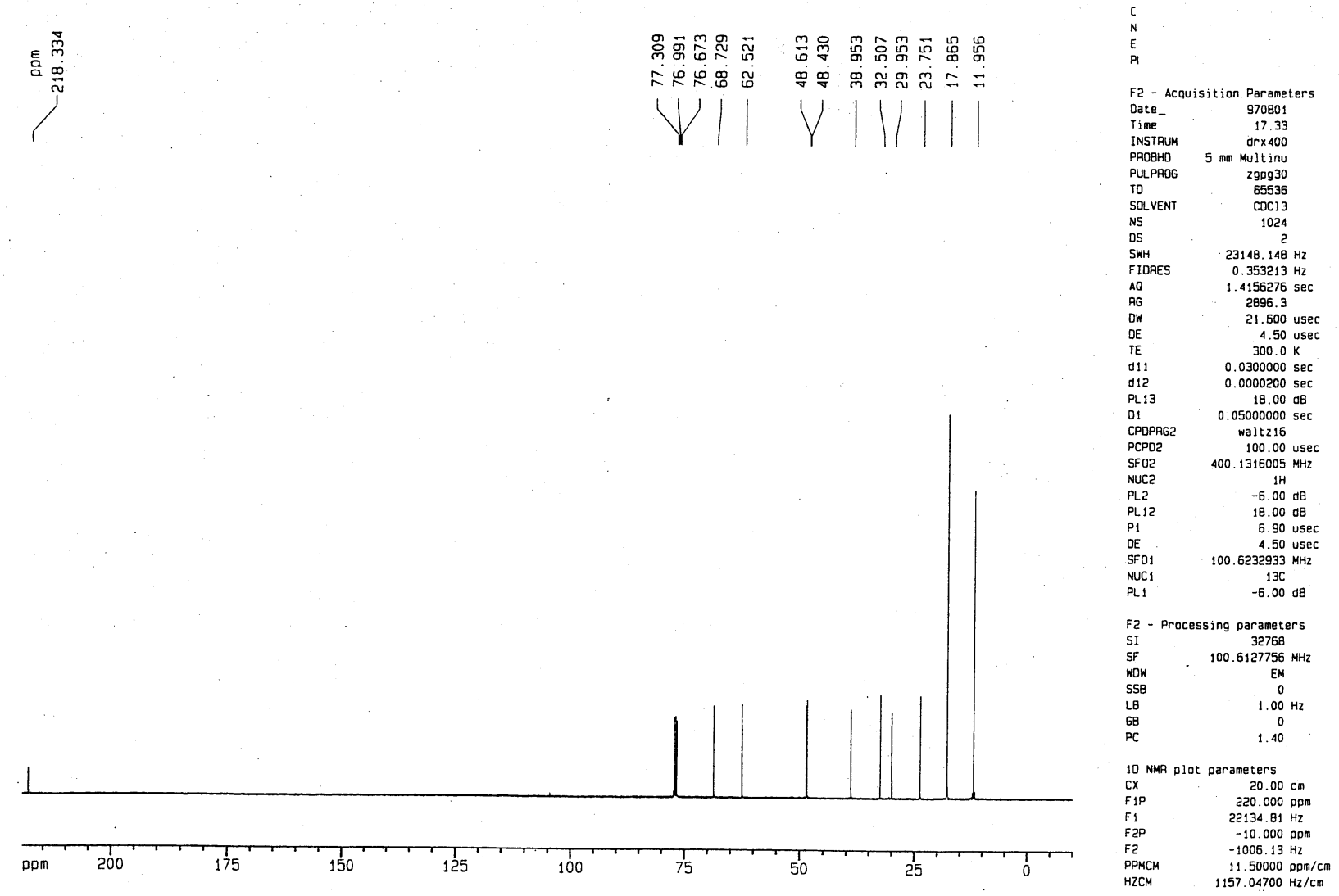

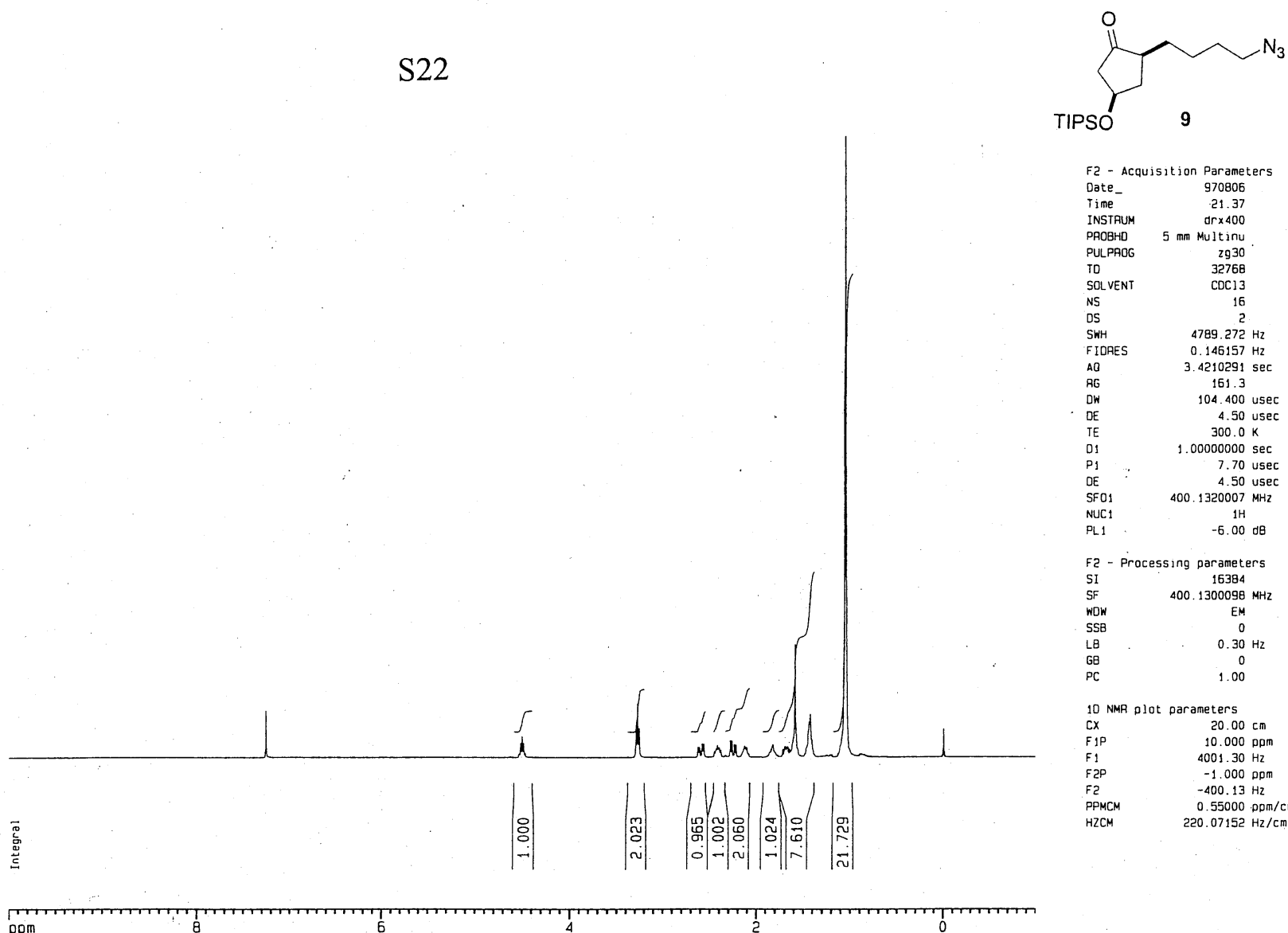

F2 - Acquisition Parameters

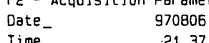

Time $\quad 21.37$

PROBHD $5 \mathrm{~mm}$ Multinu

$\begin{array}{lr}\text { PULPAOG } & 2930 \\ \text { TD } & 32768\end{array}$

$\begin{array}{ll}\text { TO } & 32368 \\ \text { SOLVENT } & \text { COC13 }\end{array}$

NS

2
$\mathrm{HHH}$$\quad 4789.272 \mathrm{~Hz}$

IDAES $\quad 0.146157 \mathrm{~Hz}$

AQ 3. $4210291 \mathrm{se}$

DW $\quad 164.400$ usec

4.50 usec

D1 $\quad 1.00000000 \mathrm{sec}$

P1 $\quad 7.70$ usec

$\begin{array}{ll}\text { OE } & 4.50 \text { usec } \\ \text { SFO1 } & 400.1320007\end{array}$

NUC1 $1 \mathrm{H}$

A Processing parameters

F2 - Processing parameters

$\begin{array}{lc}\text { SI } & 16384 \\ \text { SF } & 400.1300098 \mathrm{MHZ} \\ \text { WOW } & \text { EM } \\ \text { SSB } & 0\end{array}$

$\begin{array}{lc}\mathrm{LSB} & 0 \\ \mathrm{~GB} & 0.30 \mathrm{~Hz}\end{array}$

10 NMP plot parameters
CX $20.00 \mathrm{~cm}$

$\begin{array}{lr}\text { CX } & 20.00 \mathrm{~cm} \\ \text { FIP } & 10.000 \mathrm{pp}\end{array}$

$\begin{array}{lr}F_{1} & 10.000 \mathrm{pp} \\ \mathrm{F} & 4001.30 \mathrm{~Hz}\end{array}$

$\begin{array}{ll}F 2 & -1.000 \mathrm{ppm} \\ \text { PPMCM } & -400.13 \mathrm{~Hz}\end{array}$

PPCM $\quad 0.55000 . \mathrm{pDm} / \mathrm{Cm}$

ppm

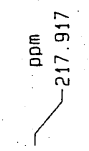

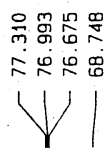

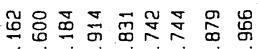

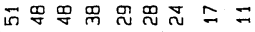
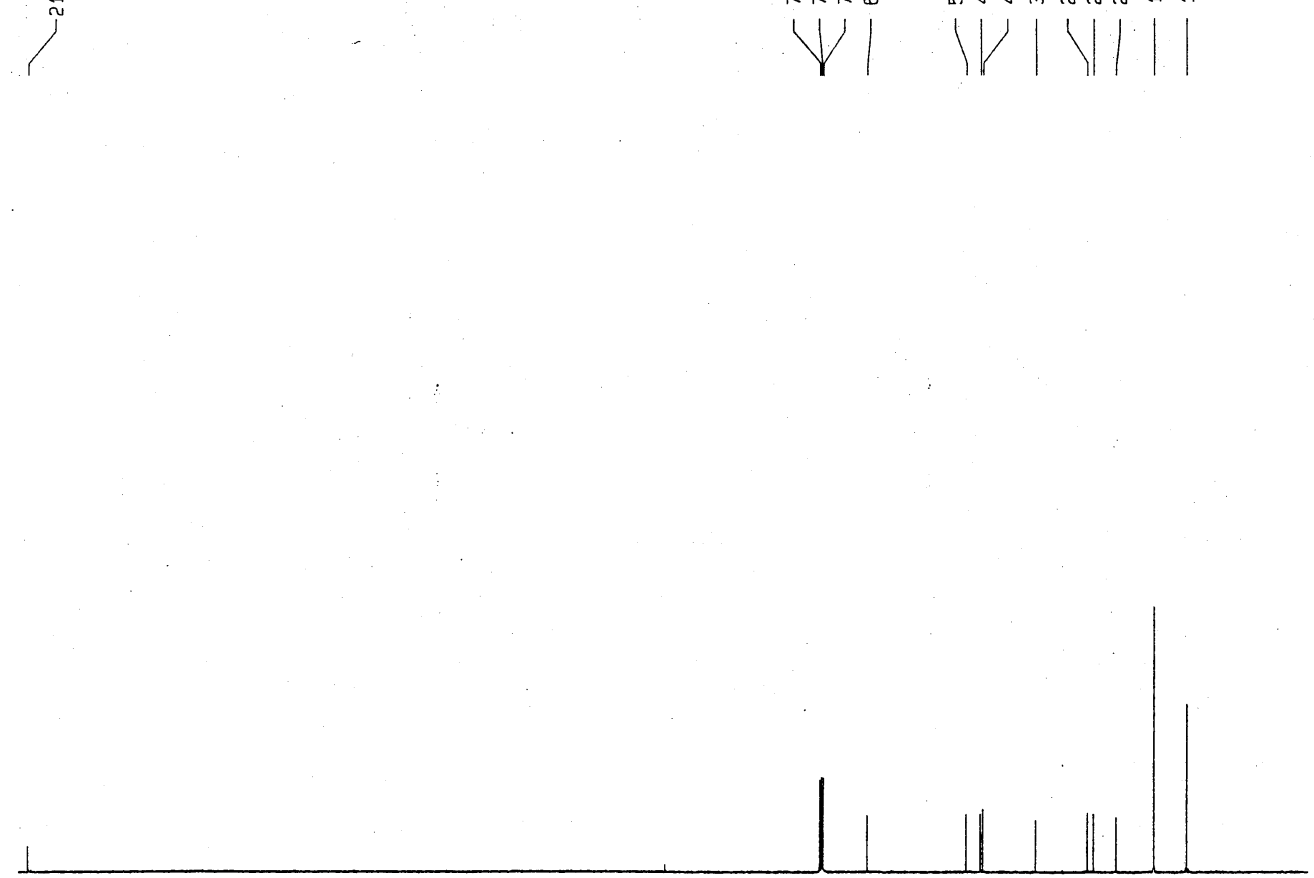

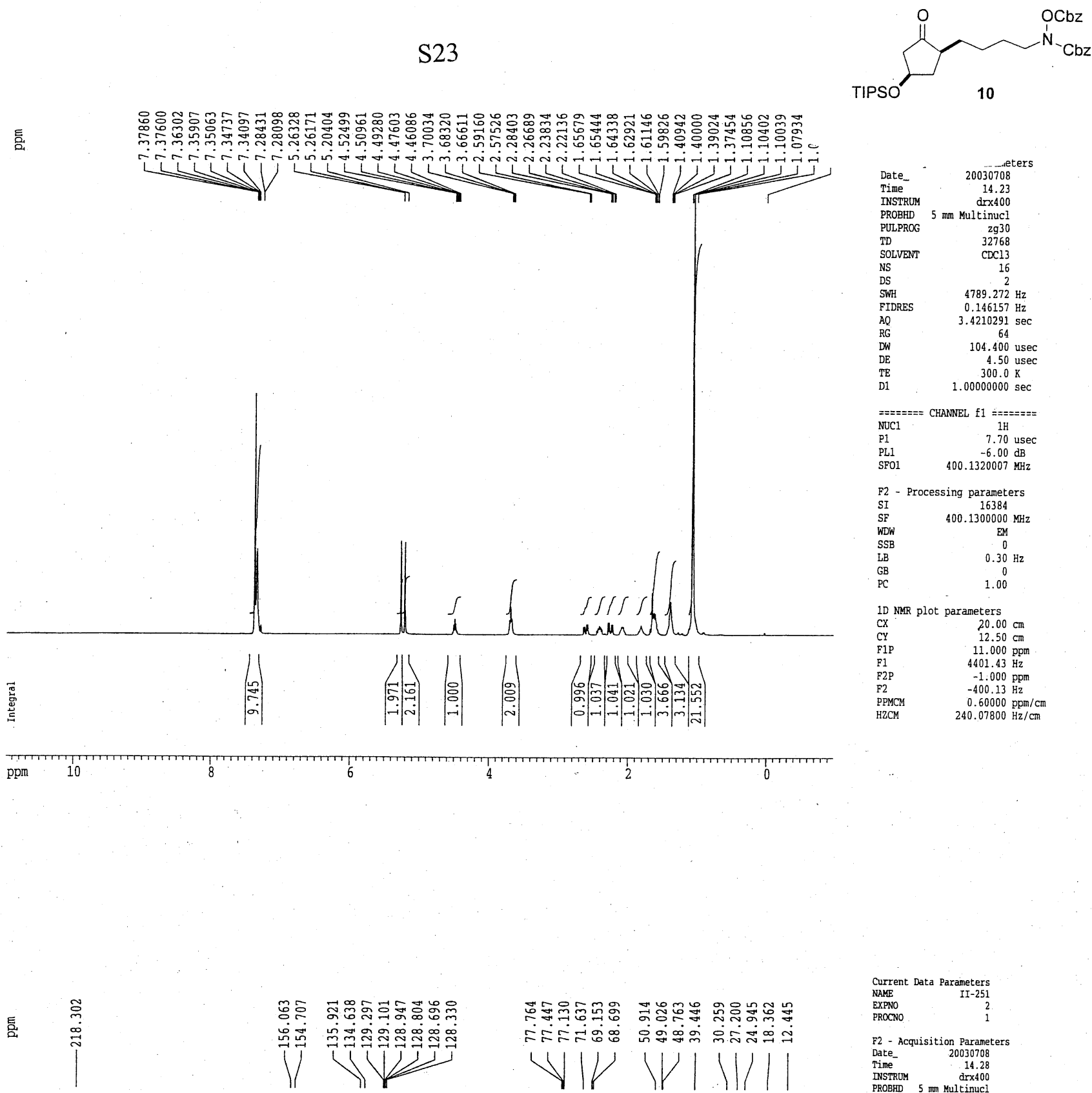

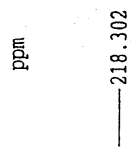

प।

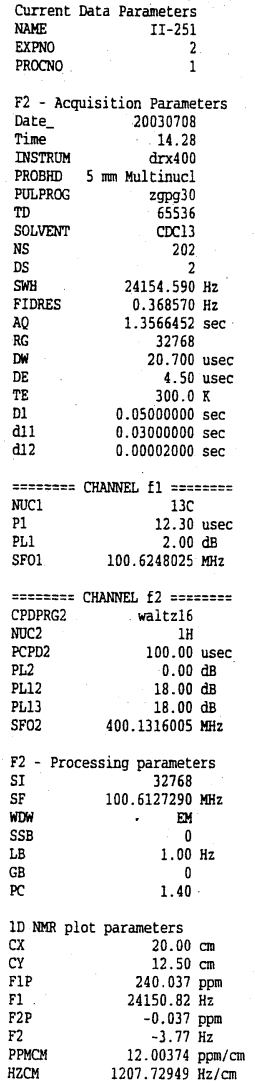




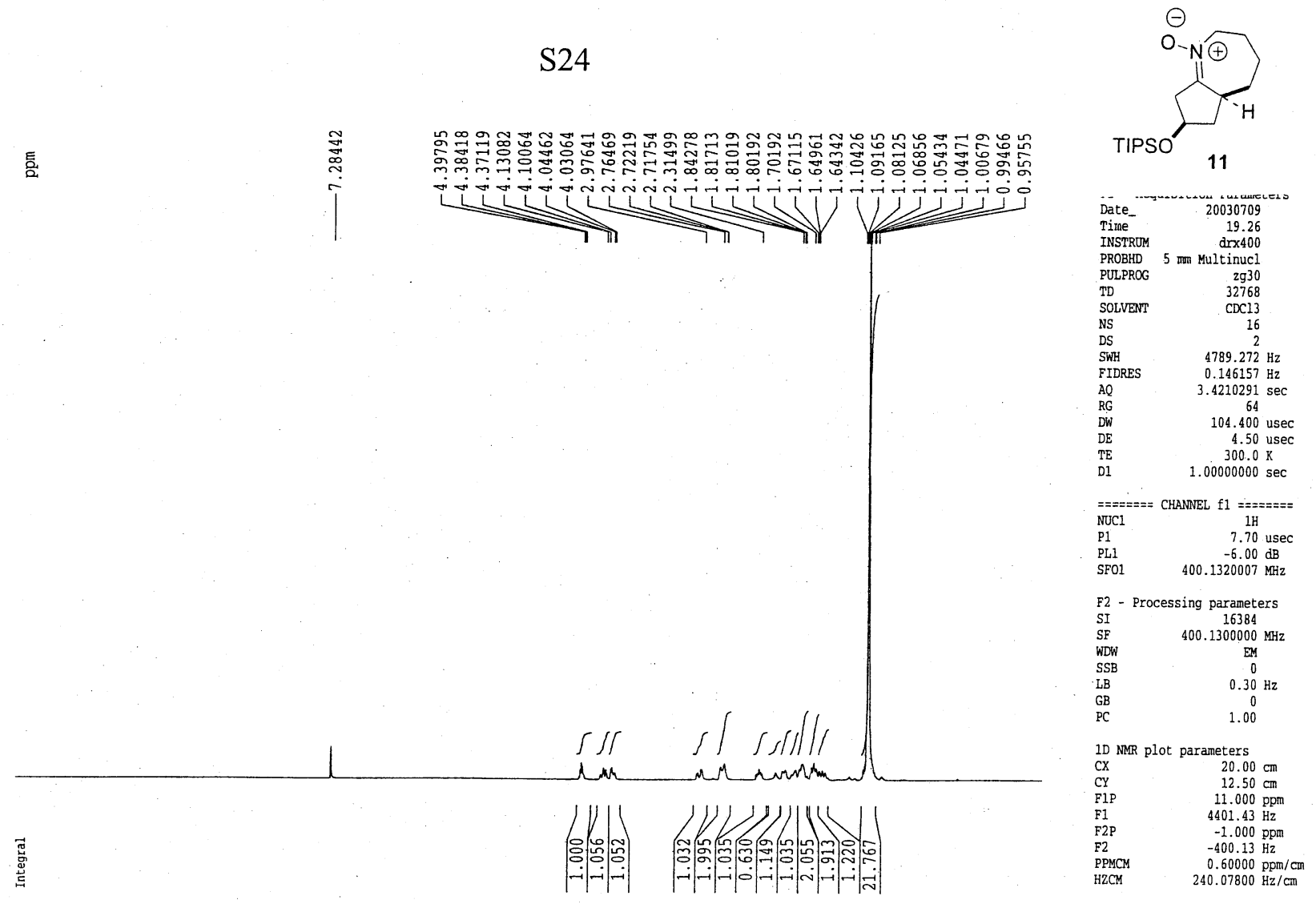

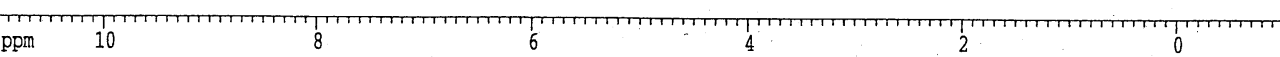

镸

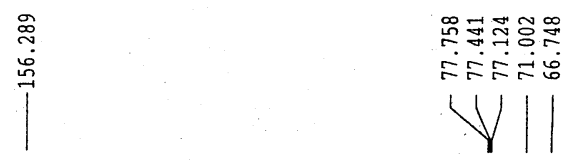

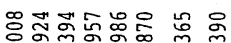

ษં丶

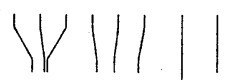

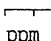

ppm 200

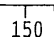

150

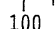

100

125

75

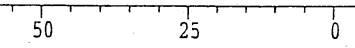



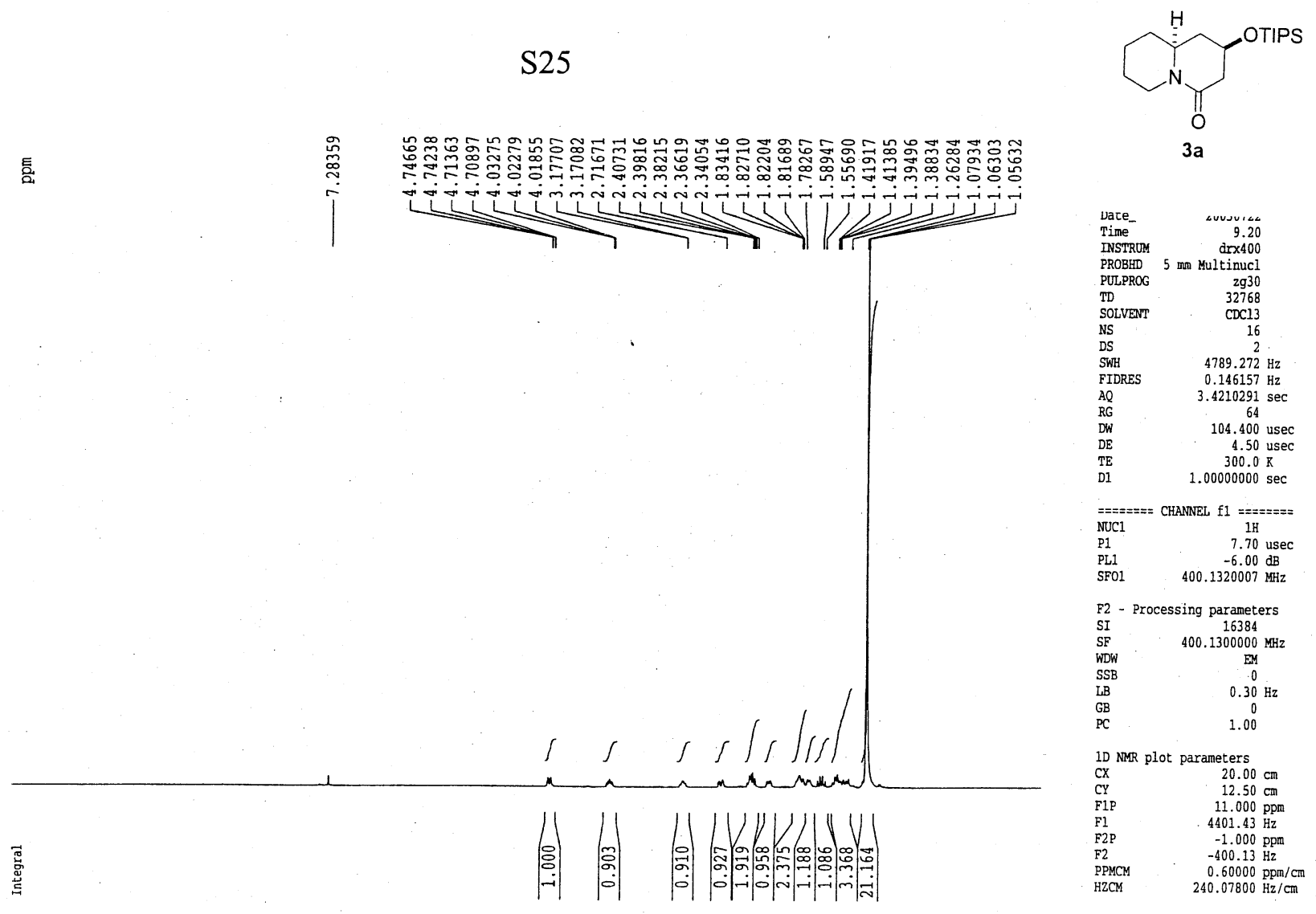

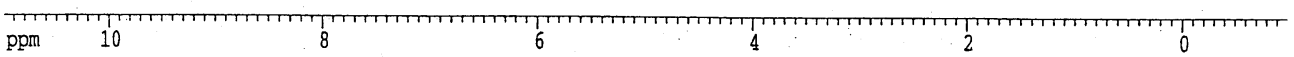

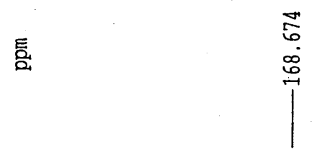

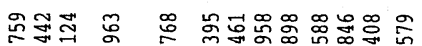

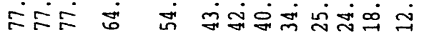
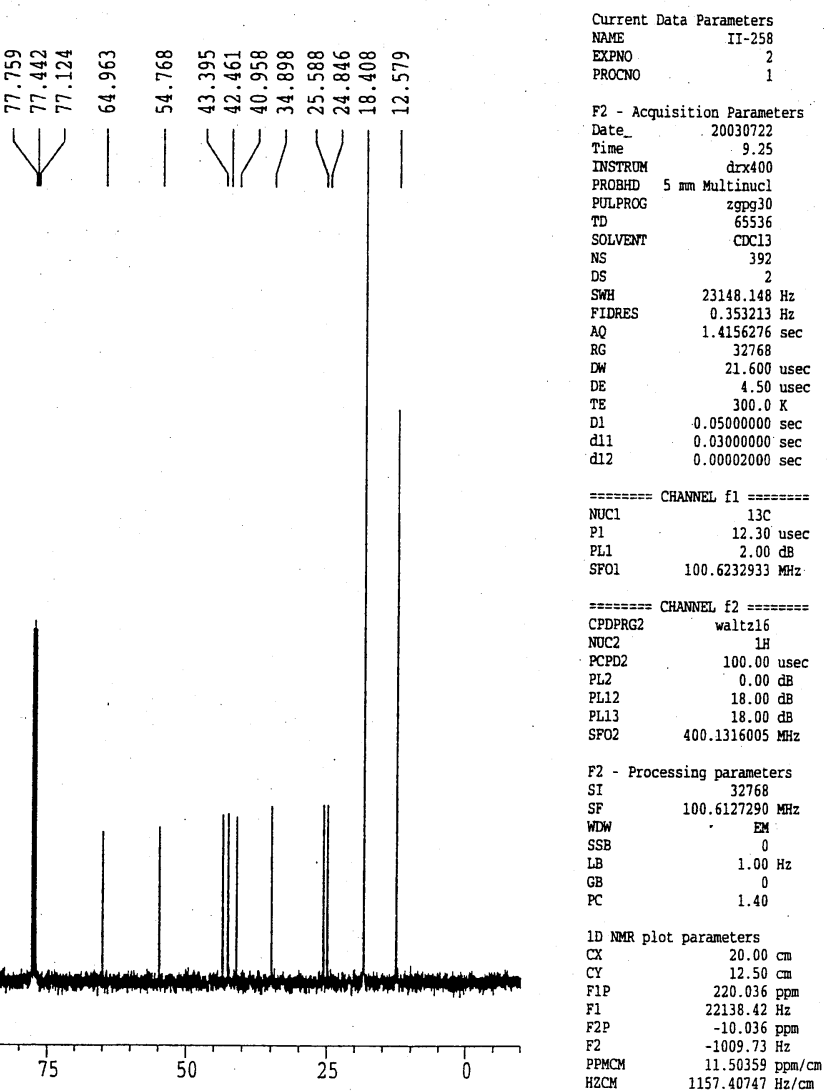

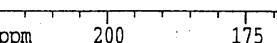

ppm

\section{(n)}

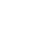




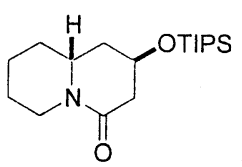

$3 b$

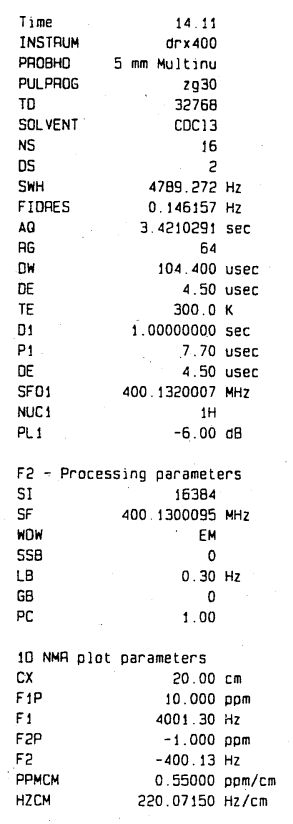

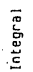

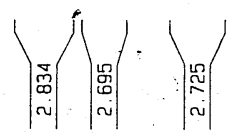

(1)

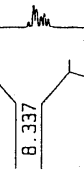<smiles>C1CCCCC1</smiles>

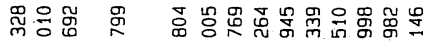

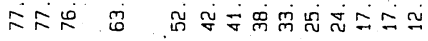

$\checkmark$

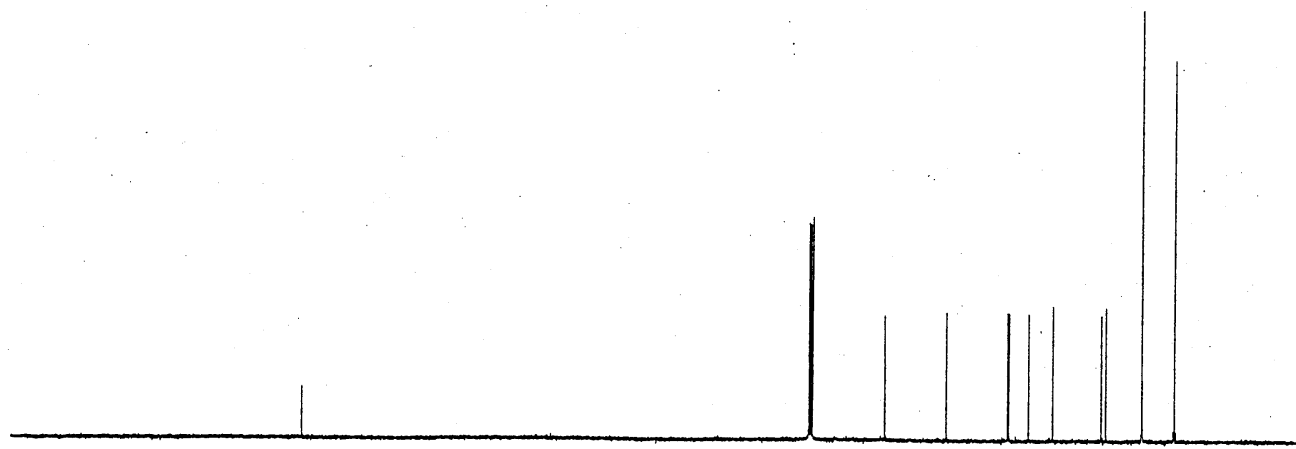



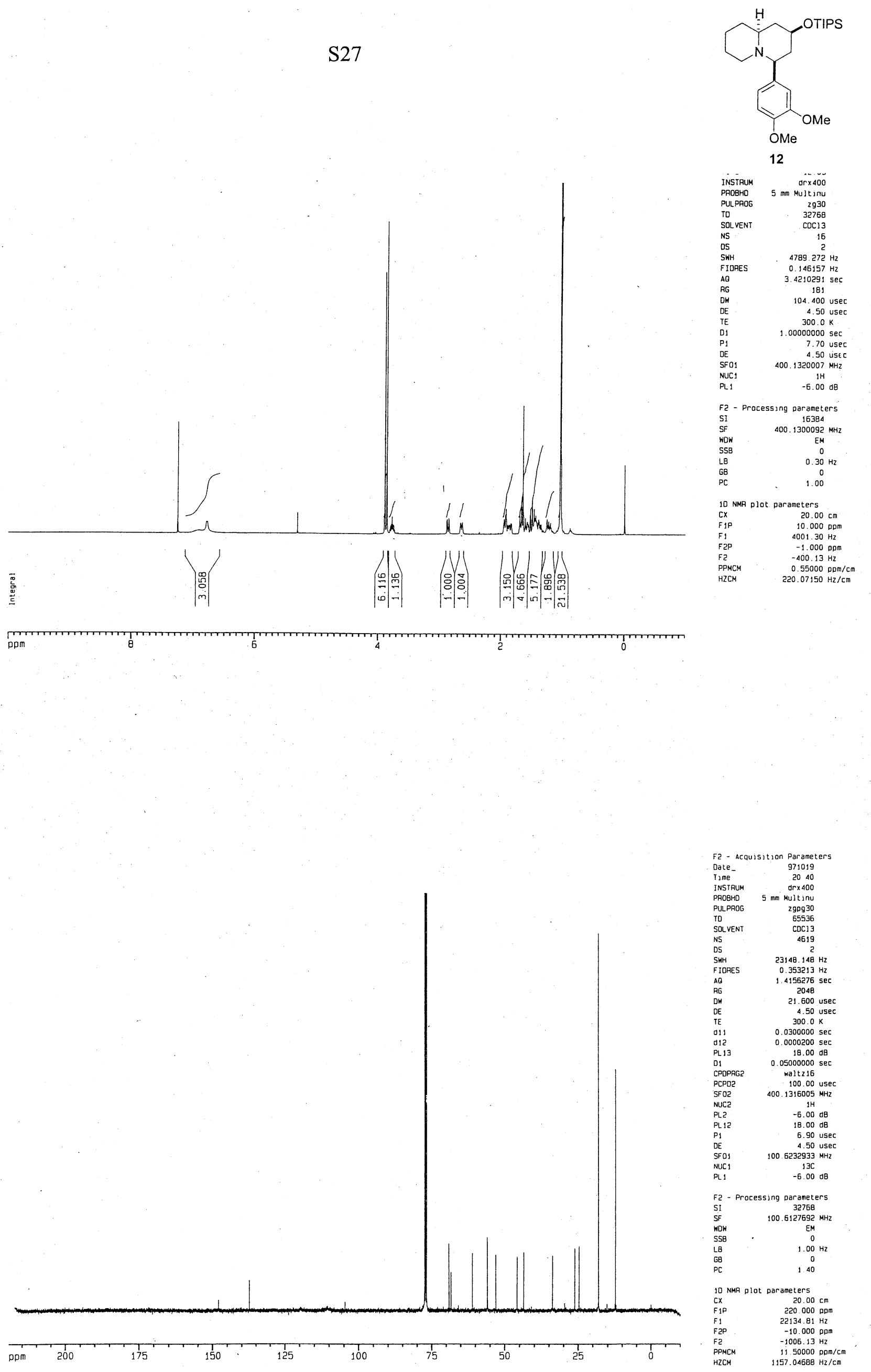

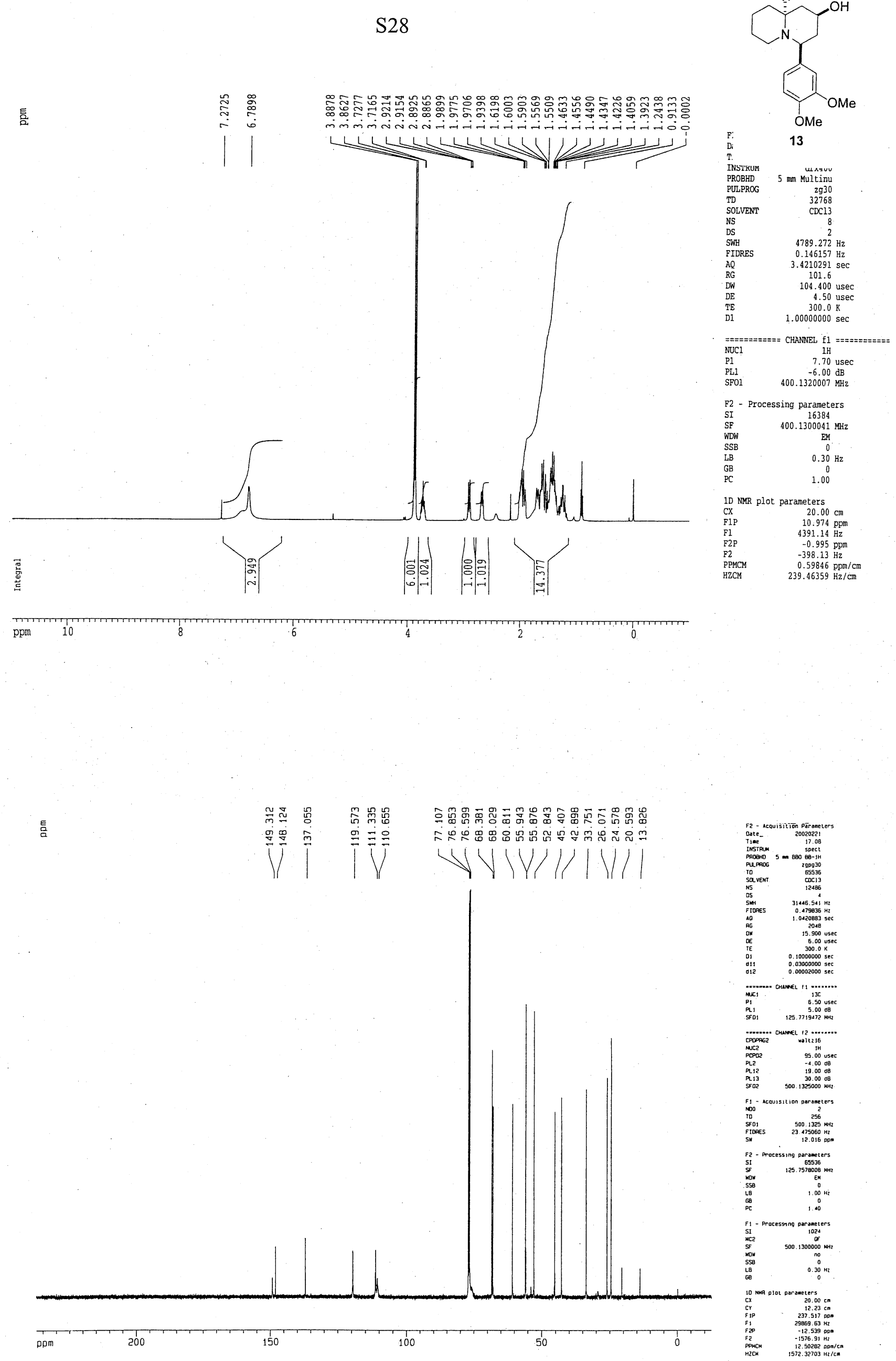


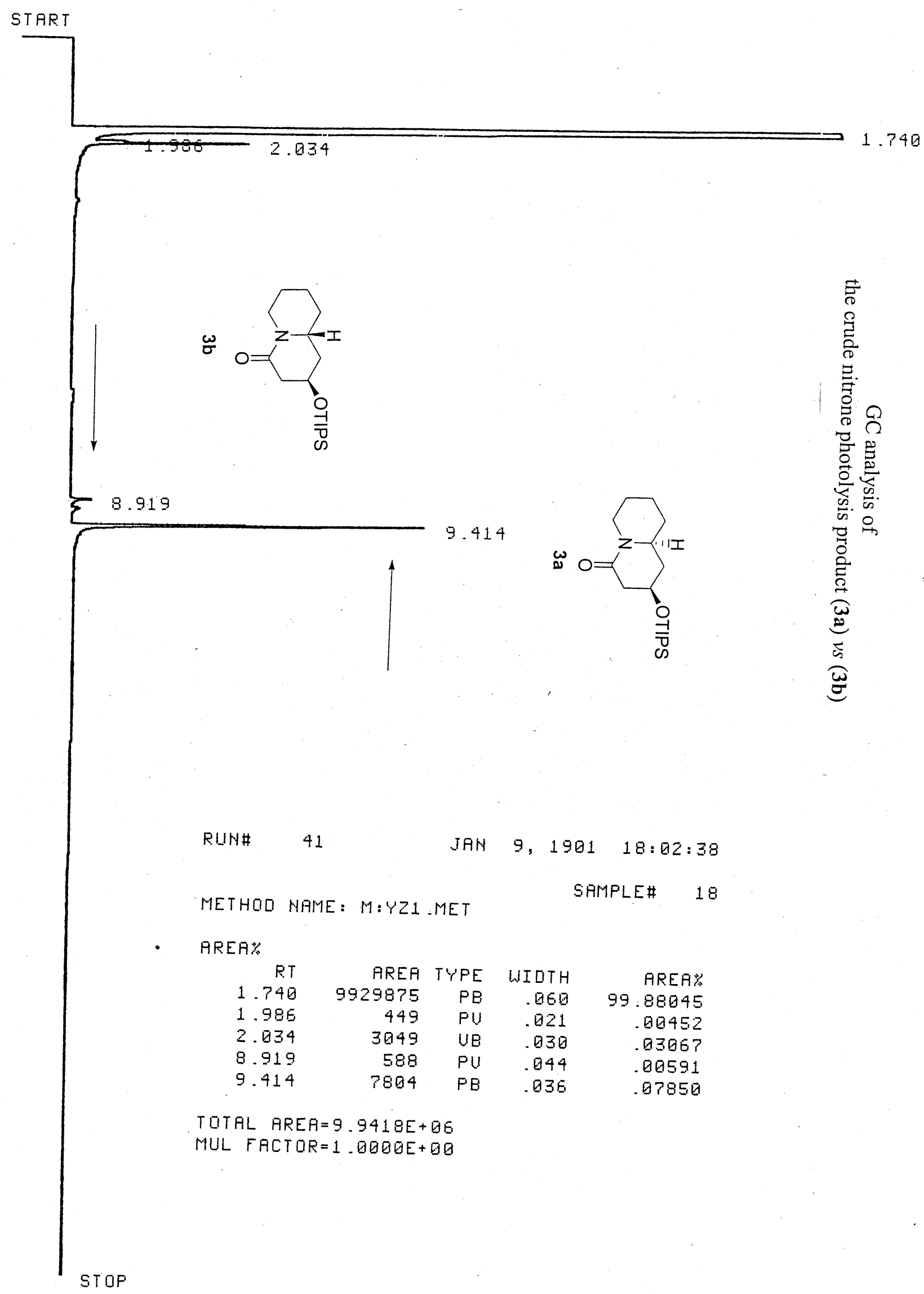




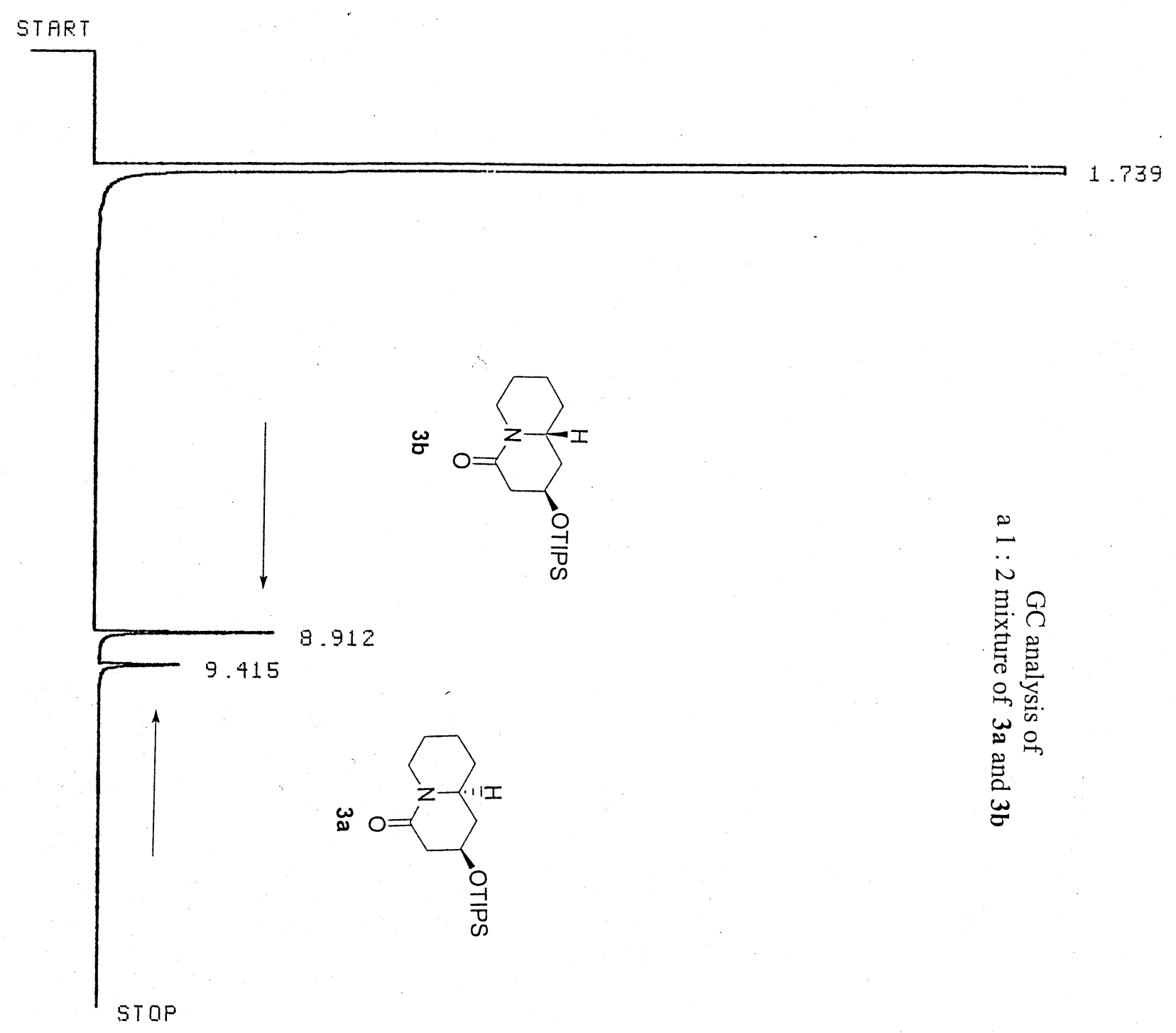

RUN\# 42 JAN $9,1901 \quad 18: 31: 59$

METHOO NAME: M:YZ1 .MET

SAMPLE\# 16

AREA:

$\begin{array}{rrrrr}\text { RT } & \text { AREA TYPE } & \text { WIDTH } & \text { AREA\% } \\ 1.739 & 9853396 & \mathrm{~PB} & .057 & 99.95184 \\ 8.912 & 3139 & \mathrm{~PB} & .036 & .03184 \\ 9.415 & 1699 & \mathrm{~PB} & .048 & .01632\end{array}$

TDTAL AREA $=9.8580 E+06$

MUL FACTOR=1. $0000 E+Q 00$ 